\title{
A SYMMETRIC NODAL CONSERVATIVE FINITE ELEMENT METHOD FOR THE DARCY EQUATION
}

\author{
GABRIEL R. BARRENECHEA, LEOPOLDO P. FRANCA ${ }^{1}$, AND FRÉDÉRIC VALENTIN ${ }^{2}$
}

\begin{abstract}
This work introduces and analyzes novel stable Petrov-Galerkin Enriched Methods (PGEM) for the Darcy problem based on the simplest but unstable continuous $\mathbb{P}_{1} / \mathbb{P}_{0}$ pair. Stability is recovered inside a Petrov-Galerkin framework where element-wise dependent residual functions, named multi-scale functions, enrich both velocity and pressure trial spaces. Unlike the velocity test space that is augmented with bubble-like functions, multiscale functions correct edge residuals as well. The multi-scale functions turn out to be the well-known lowest order Raviart-Thomas basis functions for the velocity and discontinuous quadratics polynomial functions for the pressure. The enrichment strategy suggests the way to recover the local mass conservation property for nodal-based interpolation spaces. We prove that the method and its symmetric version are well-posed and achieve optimal error estimates in natural norms. Numerical validations confirm claimed theoretical results.
\end{abstract}

\section{INTRODUCTION}

The Darcy equation arising in a porous media field belongs to the family of mixed problems [13] for which numerical methods are limited by the choice of pair of approximation spaces. From classical stable elements as the Raviart-Thomas family $\left(\mathrm{RT}_{k}\right)$ [27], Brezzi-DouglasMarini elements $\left(\mathrm{BDM}_{k}\right)$ [12], high order stable elements given in $[25,5,6]$ to more recent stabilized or least square finite element methods $[26,23,14,10,11]$ the range of possibilities to tackle the Darcy equation has increased over the past years. Methods for this problem should combine stability and accuracy while preserving physical properties inherited from the continuous problem. Properties that are only fulfilled by few of them.

To the best of our knowledge, symmetric stable nodal based finite element methods for the Darcy equation preserving mass locally remain an open problem (see [11] for a recent discussion). For example, least-squares finite element methods (cf. [10]) lead to a symmetric positive-definite system, but, in their original nodal version they are not locally mass conservative, and in [11] nodal unknowns for the velocity are forbidden. Furthermore, in

\footnotetext{
Key words and phrases. Darcy model; Enriched space; simplest element; Petrov-Galerkin approach.

${ }^{1}$ This author is supported by NSF/USA No. 0610039.

${ }^{2}$ This author is supported by CNPq /Brazil Grant No. 306255/2008-1 and 304051/2006-3, FAPERJ/Brazil Grant No. E-26/100.519/2007, and by NSF/USA No. 0610039.
} 
both cases, the lowest order piecewise constant space for the pressure is not allowed. This possibility is considered in [25], but the degrees of freedom for the velocity are not nodal.

Some of the previous requirements are satisfied by the so-called Petrov-Galerkin Enriched Methods (PGEM). PGEM have been developed in $[19,20,21]$ and further analyzed in $[18,4]$. The method is constructed by enriching polynomial functions with two types of enhancement: we add bubble functions to the test function and we add a special function to the trial function. The latter depends on the residual of the polynomial part over edges, and thus, it is no longer a bubble-like function. This gives us a Petrov-Galerkin framework. To get stabilized method forms of PGEM we use static condensation, thanks to the use of bubbles as test functions [3,8]. Number and type of degrees of freedom stay unchanged whereas basis functions incorporate unsolved sub-scales modifying their form yet preserving the polynomial basis function support. Interested readers can find a review on the subject in $[2]$.

When applied to the Darcy equation, the Petrov-Galerkin approach leads to different finite element methods [7]. One of them is obtained by searching the velocity solution into a subspace of the Raviart-Thomas space built with the Raviart-Thomas interpolation over the linear continuous trial functions. The space for the pressure stays untouched. The underlying PGEM appears to be stable for the simplest pair of interpolation spaces $\mathbb{P}_{1} / \mathbb{P}_{0}$ while preserving the mass-conservative feature, a desirable property for porous media practitioners. Performance of PGEM over several numerical tests given in [7] attests its stability and accuracy while keeping loss of local mass negligible.

Based on the previous considerations, the current work introduces a variant of the strategy proposed in [7] and leads to a final method which is symmetric, locally mass conservative and whose degrees of freedom are piecewise constants for the pressure and nodal values for the velocity. Indeed, we keep the trial space for the velocity and pressure as in [7], but the test space is built differently: it is first mapped using the Raviart-Thomas interpolation operator and then enhanced with bubble functions, an approach which allows the static condensation procedure. This new perspective opens the door to two new finite element methods, one of them fulfilling all the requirements of symmetry, nodal degrees of freedom for the velocity and locally mass conservative. Both methods prove to be well-posed and achieve optimal error estimates in natural norms. Since the starting point of our approach is a Petrov-Galerkin method, the terminology PGEM is still used in this work, even if the final methods differ from the ones presented in [7]. Finally, the approach suggests a general way of rendering some finite element methods locally mass conservative.

We end this introduction by summarizing the plan and main results of this paper: 
- In Section 2 we introduce the new finite element methods, namely, the non-symmetric (13) and its symmetric counterpart (16). We then derive the methods in a PGEM framework in Section 2.1, and in Section 2.2 we explicit the local problems solved by our enrichment basis functions (cf. (35) and (37)). In particular, we recover the local basis of the $R T_{0}$ space as the solution of (35)-(36). Finally, in Section 2.3 we prove that the discrete enhanced solution is locally mass conservative;

- Section 3 is devoted to the error analysis. We analyze in detail method (16) (the analysis of (13) is treated in Theorem 9, Section 3.3). Well posedeness and consistency error are proved first (cf. Lemmas 3 and 4) followed by convergence results (see Theorems 7 and 8 in Section 3.2). Furthermore, we use the characterization of the $R T_{0}$ interpolation operator as the solution of (35)-(36) to obtain an alternative proof for the classical $R T_{0}$ error estimate (see Corollary 6);

- The numerical tests are in Section 4 where two analytical solutions confirm theoretical results;

- Conclusions and future perspectives are drawn in Section 5;

- Finally, we relax the assumption on the source term $g$ (initially assumed piecewise constant) to propose in Appendix A an error estimate for a smooth datum $g$ (cf. Theorem 10).

1.1. Some notations. This section introduces definitions and notations used throughout. In what follows, $\Omega$ denotes an open bounded domain in $\mathbb{R}^{2}$ with polygonal boundary $\partial \Omega$, and $\mathbf{x}=\left(x_{1}, x_{2}\right)$ is a typical point in $\Omega$. As usual, $L^{2}(\Omega)$ is the space of square integrable functions over $\Omega, L_{0}^{2}(\Omega)$ represents functions belonging to $L^{2}(\Omega)$ with zero average in $\Omega$, and $H^{d i v}(\Omega)$ is composed by functions that belong to $L^{2}(\Omega)^{2}$ with divergence in $L^{2}(\Omega)$. The space $H_{0}^{d i v}(\Omega)$ stands for the space of functions belonging to $H^{d i v}(\Omega)$ which have normal component vanishing on $\partial \Omega$.

From now on we denote by $\left\{\mathcal{T}_{h}\right\}$ a family of regular triangulations of $\bar{\Omega}$ built up using triangles $K$ with boundary $\partial K$ composed by edges $F$. The set of internal edges of the triangulation $\mathcal{T}_{h}$ is denoted by $\mathcal{E}_{h}$. The characteristic length of $K$ and $F$ are denoted by $h_{K}$ and $h_{F}$, respectively, and $h:=\max \left\{h_{K}: K \in \mathcal{T}_{h}\right\}>0$, and due to the mesh regularity there exists a positive constant $C$ such that $h_{F} \leq h_{K} \leq C h_{F}$, for all $F \subseteq \partial K$. Also, for each $F=K \cap K^{\prime} \in \mathcal{E}_{h}$ we choose, once and for all, an unit normal vector $\boldsymbol{n}$ which coincides with the unit outward normal vector when $F \subseteq \partial \Omega$. The standard outward normal vector at the edge $F$ with respect to the element $K$ is denoted by $\boldsymbol{n}_{F}^{K}$. Moreover, for a function $q$, one 
denotes $\llbracket q \rrbracket$ its jump, defined by (see Figure 1$)$ :

$$
\llbracket q \rrbracket(\mathbf{x}):=\lim _{\delta \rightarrow 0^{+}} q(\mathbf{x}+\delta \boldsymbol{n})-\lim _{\delta \rightarrow 0^{-}} q(\mathbf{x}+\delta \boldsymbol{n}),
$$

and $\llbracket q \rrbracket=0$ if $F \subseteq \partial \Omega$.

Next, we denote by $H_{0}^{d i v}(K)$ the space whose functions belong to $H_{0}^{\text {div }}(\Omega)$ with support in $K$ and vanishing normal component on $\partial K$, and $L_{0}^{2}(K)$ the space of functions which belong to $L_{0}^{2}(\Omega)$ with support and zero mean in $K$. Then, we can define the corresponding global spaces

$$
H_{0}^{d i v}\left(\mathcal{T}_{h}\right):=\oplus \sum_{K \in \mathcal{T}_{h}} H_{0}^{d i v}(K) \quad \text { and } \quad L_{0}^{2}\left(\mathcal{T}_{h}\right):=\oplus \sum_{K \in \mathcal{T}_{h}} L_{0}^{2}(K)
$$

Finally, $(\cdot, \cdot)_{D}$ stands for the inner product in $L^{2}(D)$ (or in $L^{2}(D)^{2}$, when necessary), and $\|\cdot\|_{s, D}\left(|\cdot|_{s, D}\right)$ the norm (seminorm) in $H^{s}(D)$ (or $H^{s}(D)^{2}$, if necessary), and $\|\cdot\|_{d i v, D}$ the norm in $H^{d i v}(D)$.

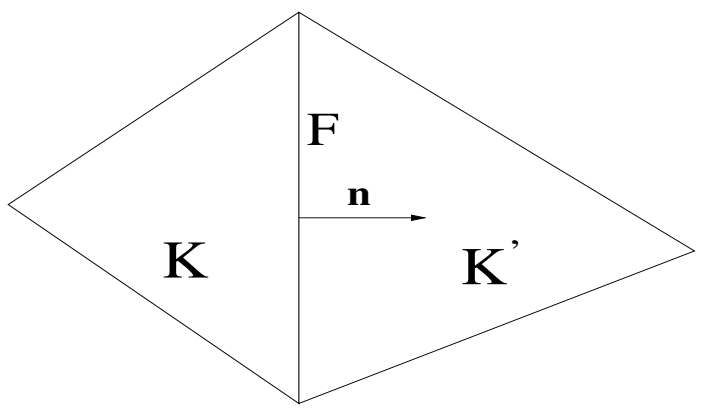

FiguRE 1. The normal vector.

1.2. Preliminaries. In this work we consider the following Darcy problem: Find $(\boldsymbol{u}, p)$ such that

$$
\begin{aligned}
\sigma \boldsymbol{u}+\nabla p=\boldsymbol{f}, \quad \nabla \cdot \boldsymbol{u}=g & \text { in } \Omega \\
\boldsymbol{u} \cdot \boldsymbol{n}=0 & \text { on } \partial \Omega,
\end{aligned}
$$

where $\sigma=\frac{\mu}{\kappa} \in \mathbb{R}^{+}$is assumed constant in $\Omega$, with $\mu$ and $\kappa$ denoting the viscosity and permeability, respectively. Here, $\boldsymbol{u}$ is the so-called Darcy velocity, $p$ is the pressure, $\boldsymbol{f}$ and $g$ are given source terms. We suppose $\boldsymbol{f}$ piecewise constant since it is usually related to the gravity force. Moreover, we assume that the given data have enough regularity and the usual compatibility condition

$$
\int_{\Omega} g=0
$$


holds.

Remark. When we consider (2) with a prescribed flux $b$ on $\partial \Omega$ such that

$$
\int_{\Omega} g=\int_{\partial \Omega} b
$$

we can recover the homogeneous case since there exists a function $\boldsymbol{w}_{b}$ belonging to $H^{\operatorname{div}}(\Omega)$ such that $\boldsymbol{w}_{b} \cdot \boldsymbol{n}=b$ on $\partial \Omega$ (cf. [22]), and thus we replace the right hand side $\boldsymbol{f}$ by $\boldsymbol{f}-\sigma \boldsymbol{w}_{b}$ and $g$ by $g-\nabla \cdot \boldsymbol{w}_{b}$.

Remark. In the more general case, $\sigma$ can always be approximated by performing projections onto the piecewise constant space. This possibility has been considered in [7]. On the other hand, despite the fact that the methods are presented in the two dimensional case, their extension to the three dimensional framework is straightforward.

The standard symmetric mixed variational formulation associated with (2) reads: Find $(\boldsymbol{u}, p) \in H_{0}^{\text {div }}(\Omega) \times L_{0}^{2}(\Omega)$ such that

$$
\mathbf{A}_{s}((\boldsymbol{u}, p),(\boldsymbol{v}, q))=\mathbf{F}_{s}(\boldsymbol{v}, q) \quad \forall(\boldsymbol{v}, q) \in H_{0}^{d i v}(\Omega) \times L_{0}^{2}(\Omega),
$$

where

$$
\mathbf{A}_{s}((\boldsymbol{u}, p),(\boldsymbol{v}, q)):=(\sigma \boldsymbol{u}, \boldsymbol{v})_{\Omega}-(p, \nabla \cdot \boldsymbol{v})_{\Omega}-(q, \nabla \cdot \boldsymbol{u})_{\Omega}, \quad \mathbf{F}_{s}(\boldsymbol{v}, q):=(\boldsymbol{f}, \boldsymbol{v})_{\Omega}-(g, q)_{\Omega}
$$

The well-posedness of (3) follows from the classical Babuska-Brezzi theory for variational problems with constraints (see [13] for more details).

Remark. An equivalent and still well-posed non-symmetric version of (3) arises from adding the weak form of the second equation to the first one in (2). The bilinear form and the linear form are now denoted by $\mathbf{A}(.,$.$) and \mathbf{F}($.$) , respectively, and are given by$

$$
\mathbf{A}((\boldsymbol{u}, p),(\boldsymbol{v}, q)):=(\sigma \boldsymbol{u}, \boldsymbol{v})_{\Omega}-(p, \nabla \cdot \boldsymbol{v})_{\Omega}+(q, \nabla \cdot \boldsymbol{u})_{\Omega}, \quad \mathbf{F}(\boldsymbol{v}, q):=(\boldsymbol{f}, \boldsymbol{v})_{\Omega}+(g, q)_{\Omega} \cdot \square
$$

Next, the classical discrete mixed formulation of this problem is: Find $\left(\boldsymbol{u}_{h}, p_{h}\right) \in \mathbf{V}_{h} \times Q_{h}$ such that

$$
\mathbf{A}_{s}\left(\left(\boldsymbol{u}_{h}, p_{h}\right),\left(\boldsymbol{v}_{h}, q_{h}\right)\right)=\mathbf{F}_{s}\left(\boldsymbol{v}_{h}, q_{h}\right) \quad \forall\left(\boldsymbol{v}_{h}, q_{h}\right) \in \mathbf{V}_{h} \times Q_{h},
$$

where $\mathbf{V}_{h}$ and $Q_{h}$ are finite-dimensional approximations of $H_{0}^{d i v}(\Omega)$ and $L_{0}^{2}(\Omega)$, respectively. It is well known that the pair of interpolation spaces for pressure and velocity must satisfy the discrete Babuska-Brezzi (or inf-sup) condition [13] in order to lead to a stable discrete version of problem (5). For the Darcy model containing a zero order term, this restriction 
has been proved to be unnecessary. In fact, in [24] standard continuous Lagrangian finite element spaces have been proved to be stable and convergent.

The lowest order Raviart-Thomas space is one of the simplest examples of a stable mass conservative element, and is composed by the velocity space

$$
\mathbf{V}_{R T_{0}}:=\left\{\boldsymbol{v} \in H_{0}^{d i v}(\Omega):\left.\boldsymbol{v}\right|_{K} \in \mathrm{RT}_{0}(K) \forall K \in \mathcal{T}_{h}\right\}
$$

where the local space $\mathrm{RT}_{0}(K)$ is defined by

$$
\operatorname{RT}_{0}(K):=\mathbb{P}_{0}(K)^{2}+\mathbf{x} \mathbb{P}_{0}(K)
$$

and

$$
Q_{h}:=\left\{q_{0} \in L_{0}^{2}(\Omega):\left.q_{0}\right|_{K} \in \mathbb{P}_{0}(K) \forall K \in \mathcal{T}_{h}\right\}
$$

Hence, only the normal component of the velocity is continuous and the inf-sup condition is satisfied since $\nabla \cdot \mathbf{V}_{R T_{0}}=Q_{h}$. Associated with the space $\mathrm{RT}_{0}(K)$ there exists a natural local interpolation operator $\pi_{K}:\left[H^{1}(K)\right]^{2} \rightarrow \mathrm{RT}_{0}(K)$, defined by (cf. [13, 17])

$$
\int_{F} \pi_{K}(\boldsymbol{v}) \cdot \boldsymbol{n}=\int_{F} \boldsymbol{v} \cdot \boldsymbol{n},
$$

for all $F \in \partial K$, or, equivalently

$$
\pi_{K}(\boldsymbol{v}):=\sum_{F \subseteq \partial K} \frac{\int_{F} \boldsymbol{v} \cdot \boldsymbol{n}}{h_{F}} \boldsymbol{\varphi}_{F},
$$

where $\boldsymbol{\varphi}_{F}$ is the Raviart-Thomas' basis function given by

$$
\boldsymbol{\varphi}_{F}(\mathbf{x})= \pm \frac{h_{F}}{2|K|}\left(\mathbf{x}-\mathbf{x}_{F}\right)
$$

and $\mathbf{x}_{F}$ denotes the node opposite to the edge $F$. Hence, a global interpolation operator noted $\pi:\left[H^{1}(\Omega)\right]^{2} \rightarrow \mathbf{V}_{R T_{0}}$ follows by defining $\left.\pi(\boldsymbol{v})\right|_{K}=\pi_{K}(\boldsymbol{v})$ in each $K \in \mathcal{T}_{h}$.

Remark. The sign before the Raviart-Thomas basis function $\boldsymbol{\varphi}_{F}$ depends on whether the normal vector $\boldsymbol{n}$ on $F \subseteq \partial K$ points inwards or outwards $K$.

A lifting operator from $L^{1}(F)$ to $\mathbf{V}_{R T_{0}}$ will be needed in the sequel, it is denoted by $\ell$ and is such that $\ell(q):=\sum_{F \in \mathcal{E}_{h}} \ell_{F}(q)$ where

$$
\ell_{F}(q)=\frac{\alpha_{F} \int_{F} q}{\sigma} \varphi_{F}
$$

where the coefficient $\alpha_{F}$ is a given positive constant which is independent of $h_{F}$ and $\sigma$, but can vary with $F$. We finally denote, for $K \in \mathcal{T}_{h}, \ell_{K}(q):=\left.\ell(q)\right|_{K}$. 


\section{The Finite ELEMENT METhodS}

We begin by introducing the standard finite element space $\mathbf{V}_{h}:=\left[V_{h}\right]^{2} \cap H_{0}^{\operatorname{div}}(\Omega)$ for the velocity variable, where

$$
V_{h}:=\left\{v \in C^{0}(\bar{\Omega}):\left.v\right|_{K} \in \mathbb{P}_{1}(K), \forall K \in \mathcal{T}_{h}\right\}
$$

whereas the pressure is discretized using the space $Q_{h}$ defined in (7). We start by presenting the methods that will be analyzed in this work. First, the Petrov-Galerkin Enriched Method reads: Find $\left(\boldsymbol{u}_{1}, p_{0}\right) \in \mathbf{V}_{h} \times Q_{h}$ such that

$$
\mathbf{B}\left(\left(\boldsymbol{u}_{1}, p_{0}\right),\left(\boldsymbol{v}_{1}, q_{0}\right)\right)=\mathbf{F}_{s}\left(\pi\left(\boldsymbol{v}_{1}\right), q_{0}\right)
$$

for all $\left(\boldsymbol{v}_{1}, q_{0}\right) \in \mathbf{V}_{h} \times Q_{h}$, where

$\mathbf{B}\left(\left(\boldsymbol{u}_{1}, p_{0}\right),\left(\boldsymbol{v}_{1}, q_{0}\right)\right):=\mathbf{A}_{s}\left(\left(\pi\left(\boldsymbol{u}_{1}\right), p_{0}\right),\left(\pi\left(\boldsymbol{v}_{1}\right), q_{0}\right)\right)+\left(\ell\left(\llbracket p_{0} \rrbracket\right), \sigma \pi\left(\boldsymbol{v}_{1}\right)\right)_{\Omega}-\sum_{F \in \mathcal{E}_{h}} \tau_{F}\left(\llbracket p_{0} \rrbracket, \llbracket q_{0} \rrbracket\right)_{F}$, and $\pi$ and $\ell$ are the operators defined through (8) and (11), respectively, and the coefficient $\tau_{F}$ stands for

$$
\tau_{F}:=\frac{\alpha_{F} h_{F}}{\sigma}
$$

In Section 3 this problem is proved to be well-posed for an appropriate choice of $\alpha_{F}$.

Alternatively, a symmetric related formulation can also be derived and reads: Find $\left(\hat{\boldsymbol{u}}_{1}, \hat{p}_{0}\right) \in$ $\mathbf{V}_{h} \times Q_{h}$ such that

$$
\mathbf{B}_{s}\left(\left(\hat{\boldsymbol{u}}_{1}, \hat{p}_{0}\right),\left(\boldsymbol{v}_{1}, q_{0}\right)\right)=\mathbf{F}_{s}\left(\pi\left(\boldsymbol{v}_{1}\right), q_{0}\right),
$$

for all $\left(\boldsymbol{v}_{1}, q_{0}\right) \in \mathbf{V}_{h} \times Q_{h}$, where

$$
\mathbf{B}_{s}\left(\left(\boldsymbol{u}_{1}, p_{0}\right),\left(\boldsymbol{v}_{1}, q_{0}\right)\right):=\mathbf{A}_{s}\left(\left(\pi\left(\boldsymbol{u}_{1}\right), p_{0}\right),\left(\pi\left(\boldsymbol{v}_{1}\right), q_{0}\right)\right)-\sum_{F \in \mathcal{E}_{h}} \tau_{F}\left(\llbracket p_{0} \rrbracket, \llbracket q_{0} \rrbracket\right)_{F} .
$$

Remark. The latter method is based on the error analysis (see §3) which points out that we can remove from $(13)$ the term $\left(\ell\left(\llbracket p_{0} \rrbracket\right), \sigma \pi\left(\boldsymbol{v}_{1}\right)\right)_{\Omega}$ without introducing a loss of accuracy. Moreover, we recover the symmetric form of the so-called reduced PGEM method presented in [7], by replacing the term $\left(\pi\left(\boldsymbol{u}_{1}\right), \pi\left(\boldsymbol{v}_{1}\right)\right)_{\Omega}$ by $\left(\boldsymbol{u}_{1}, \boldsymbol{v}_{1}\right)_{\Omega}$ in (13). This reduced method turns out to be optimally convergent [14], and in $§ 2.3$ we show how to render it locally mass conservative. 
2.1. Derivation of the methods. The starting point towards our final method is the following Petrov-Galerkin method for (2): Find $\boldsymbol{u}_{h}:=\boldsymbol{u}_{1}+\boldsymbol{u}_{e} \in \mathbf{V}_{h}+H_{0}^{\operatorname{div}}(\Omega)$ and $p_{h}:=$ $p_{0}+p_{e} \in Q_{h} \oplus L_{0}^{2}\left(\mathcal{T}_{h}\right)$ such that

$$
\mathbf{A}_{s}\left(\left(\boldsymbol{u}_{h}, p_{h}\right),\left(\boldsymbol{v}_{h}, q_{h}\right)\right)=\mathbf{F}_{s}\left(\boldsymbol{v}_{h}, q_{h}\right)
$$

for all $\boldsymbol{v}_{h}:=\pi\left(\boldsymbol{v}_{1}\right)+\boldsymbol{v}_{b} \in \pi\left(\mathbf{V}_{h}\right) \oplus H_{0}^{d i v}\left(\mathcal{T}_{h}\right)$ and for all $q_{h}:=q_{0}+q_{e} \in Q_{h} \oplus L_{0}^{2}\left(\mathcal{T}_{h}\right)$. Here $\pi\left(\mathbf{V}_{h}\right)$ stands for the subspace of $\mathbf{V}_{R T_{0}}$ built as the image of space $\mathbf{V}_{h}$ through the operator $\pi$.

This scheme is equivalent to the following system: for all $\left(\boldsymbol{v}_{1}, q_{0}\right) \in \mathbf{V}_{h} \times Q_{h}$ and for all $\left(\boldsymbol{v}_{b}, q_{e}\right) \in H_{0}^{\operatorname{div}}\left(\mathcal{T}_{h}\right) \times L_{0}^{2}\left(\mathcal{T}_{h}\right)$

$$
\begin{aligned}
\mathbf{A}_{s}\left(\left(\boldsymbol{u}_{h}, p_{h}\right),\left(\pi\left(\boldsymbol{v}_{1}\right), q_{0}\right)\right) & =\mathbf{F}_{s}\left(\pi\left(\boldsymbol{v}_{1}\right), q_{0}\right), \\
\mathbf{A}_{s}\left(\left(\boldsymbol{u}_{h}, p_{h}\right),\left(\boldsymbol{v}_{b}, q_{e}\right)\right) & =\mathbf{F}_{s}\left(\boldsymbol{v}_{b}, q_{e}\right)
\end{aligned}
$$

From now on, and just in order to derive the method, we will assume that $g$ is a piecewise constant function (even if the method is analyzed and implemented for more general functions $g$ ). With this assumption in mind, starting from (20) and proceeding as in [7], the following strong problem is obtained for $\left(\boldsymbol{u}_{e}, p_{e}\right)$ :

$$
\begin{gathered}
\sigma \boldsymbol{u}_{e}+\nabla p_{e}=\boldsymbol{f}-\sigma \boldsymbol{u}_{1}, \quad \nabla \cdot \boldsymbol{u}_{e}=\overline{\llbracket p_{0} \rrbracket}+\left(\Pi_{K}\left(\nabla \cdot \boldsymbol{u}_{1}\right)-\nabla \cdot \boldsymbol{u}_{1}\right) \quad \text { in } K \\
\sigma \boldsymbol{u}_{e} \cdot \boldsymbol{n}=\alpha_{F} \int_{F} \llbracket p_{0} \rrbracket+\left(\left(\boldsymbol{f}-\sigma \boldsymbol{u}_{1}\right) \cdot \boldsymbol{n}-\Pi_{F}\left(\left(\boldsymbol{f}-\sigma \boldsymbol{u}_{1}\right) \cdot \boldsymbol{n}\right)\right)
\end{gathered}
$$

on each $F \subseteq \partial K \cap \mathcal{E}_{h}$, and $\boldsymbol{u}_{e} \cdot \boldsymbol{n}=0$ on $F \subseteq \partial \Omega$. Here, $\Pi_{F}$ and $\Pi_{K}$ stand for the $L^{2}$ projection operators over the constant space, i.e, $\Pi_{F}(v)=\frac{1}{h_{F}} \int_{F} v$ and $\Pi_{K}(v)=\frac{1}{|K|} \int_{K} v$. Finally, the constant $\overline{\llbracket p_{0} \rrbracket}$ is chosen in order to make (21)-(22) compatible, and is given by

$$
\overline{\llbracket p_{0} \rrbracket}:=\frac{1}{|K|} \sum_{i=1}^{3} \frac{\alpha_{F_{i}} h_{F_{i}}}{\sigma} \int_{F_{i}} \llbracket p_{0} \rrbracket \boldsymbol{n} \cdot \boldsymbol{n}_{F_{i}}^{K} .
$$

Remark. For higher order velocity interpolation the vanishing right hand side term $\Pi_{K}(\nabla$. $\left.\boldsymbol{u}_{1}\right)-\nabla \cdot \boldsymbol{u}_{1}$ left in the local problem (21) needs to be taken into account. Moreover, written in this form, it will help us to bound the consistency errors (see equation (62) below). On the other hand, since we have assumed that $\boldsymbol{f}$ is a constant (or piecewise constant) function, the divergence equation in (21) may be rewritten as follows

$$
\nabla \cdot \boldsymbol{u}_{e}=\overline{\llbracket p_{0} \rrbracket}+\frac{1}{\sigma}\left(\nabla \cdot\left(\boldsymbol{f}-\sigma \boldsymbol{u}_{1}\right)-\Pi_{K}\left(\nabla \cdot\left(\boldsymbol{f}-\sigma \boldsymbol{u}_{1}\right)\right)\right)
$$

which is the form that we will consider from now on. 
Remark. The boundary condition (22) assures the continuity of the normal component of the velocity on each edge, thus keeping our approach conforming. This fact may be kept even for discontinuous coefficients, as it has been done in [7], where the mean value of $\sigma$ has been included in the boundary condition.

Now, let $\mathcal{M}_{K}:=\left(\mathcal{M}_{K}^{u}, \mathcal{M}_{K}^{p}\right): H^{1}(K)^{2} \rightarrow H_{0}^{\text {div }}(K) \times L_{0}^{2}(K)$ and $\mathcal{D}_{K}:=\left(\mathcal{D}_{K}^{u}, \mathcal{D}_{K}^{p}\right):$ $L^{2}(\partial K) \rightarrow H^{d i v}(K) \times L_{0}^{2}(K)$, defined as follows: $\left(\boldsymbol{v}_{e}, \eta_{e}\right):=\left(\mathcal{M}_{K}^{u}(\boldsymbol{v}), \mathcal{M}_{K}^{p}(\boldsymbol{v})\right)$ is the solution of

$$
\begin{aligned}
\sigma \boldsymbol{v}_{e}+\nabla \eta_{e} & =\boldsymbol{v}, \quad \sigma \nabla \cdot \boldsymbol{v}_{e}=\nabla \cdot \boldsymbol{v}-\Pi_{K}(\nabla \cdot \boldsymbol{v}) \text { in } K, \\
\sigma \boldsymbol{v}_{e} \cdot \boldsymbol{n} & =\boldsymbol{v} \cdot \boldsymbol{n}-\Pi_{F}(\boldsymbol{v} \cdot \boldsymbol{n}) \quad \text { on } \quad \text { each } F \subseteq \partial K
\end{aligned}
$$

and $\left(\boldsymbol{w}_{e}, \xi_{e}\right):=\left(\mathcal{D}_{K}^{u}(q), \mathcal{D}_{K}^{p}(q)\right)$ solves

$$
\begin{gathered}
\sigma \boldsymbol{w}_{e}+\nabla \xi_{e}=\mathbf{0}, \quad \nabla \cdot \boldsymbol{w}_{e}=\frac{1}{|K|} \sum_{i=1}^{3} \frac{\alpha_{F_{i}} h_{F_{i}}}{\sigma} \int_{F_{i}} q \boldsymbol{n} \cdot \boldsymbol{n}_{F_{i}}^{K} \text { in } K \\
\sigma \boldsymbol{w}_{e} \cdot \boldsymbol{n}=\alpha_{F} \int_{F} q \text { on each } F \subseteq \partial K \cap \Omega .
\end{gathered}
$$

Then, using these operators and (23), we can characterize the solution $\left(\boldsymbol{u}_{e}, p_{e}\right)=\left(\boldsymbol{u}_{e}^{M}+\right.$ $\left.\boldsymbol{u}_{e}^{D}, p_{e}^{M}+p_{e}^{D}\right)$ of $(21)-(22)$ as follows

$$
\begin{gathered}
\left(\boldsymbol{u}_{e}^{M}, p_{e}^{M}\right)=\mathcal{M}_{K}\left(\boldsymbol{f}-\sigma \boldsymbol{u}_{1}\right) \quad \forall K \in \mathcal{T}_{h}, \\
\left(\boldsymbol{u}_{e}^{D}, p_{e}^{D}\right)=\mathcal{D}_{K}\left(\llbracket p_{0} \rrbracket\right) \quad \forall K \in \mathcal{T}_{h} .
\end{gathered}
$$

Next, we turn back to equation (19). First, since $p_{e} \in L_{0}^{2}(K)$ and $\left.\nabla \cdot \pi\left(\boldsymbol{v}_{1}\right)\right|_{K} \in \mathbb{R}$ we obtain

$$
\left(p_{e}, \nabla \cdot \pi\left(\boldsymbol{v}_{1}\right)\right)_{K}=0 \quad \text { for all } K \in \mathcal{T}_{h}
$$

Therefore, the problem (19) becomes: Find $\left(\boldsymbol{u}_{1}, p_{0}\right) \in \mathbf{V}_{h} \times Q_{h}$ such that

$$
\mathbf{A}_{s}\left(\left(\boldsymbol{u}_{1}+\boldsymbol{u}_{e}, p_{0}\right),\left(\pi\left(\boldsymbol{v}_{1}\right), q_{0}\right)\right)=\mathbf{F}_{s}\left(\pi\left(\boldsymbol{v}_{1}\right), q_{0}\right) \quad \forall\left(\boldsymbol{v}_{1}, q_{0}\right) \in \mathbf{V}_{h} \times Q_{h}
$$

where $\boldsymbol{u}_{e}$ is characterized with respect to $\boldsymbol{u}_{1}$ and $p_{0}$ by (26)-(27). It is also convenient to rewrite the problem above in an equivalent form integrating it by parts in each $K \in \mathcal{T}_{h}$

$$
\sum_{K \in \mathcal{T}_{h}}\left(q_{0}, \nabla \cdot \boldsymbol{u}_{e}\right)_{K}=\sum_{F \in \mathcal{E}_{h}} \tau_{F}\left(\llbracket p_{0} \rrbracket, \llbracket q_{0} \rrbracket\right)_{F} .
$$


Remark. The term related to $\boldsymbol{f}$ in (26) vanishes. Indeed, since $\boldsymbol{f}$ is constant in $K$ then $\boldsymbol{f} \cdot \boldsymbol{n}-\Pi_{F}(\boldsymbol{f} \cdot \boldsymbol{n})=0$ on each edge $F$, and there also exists a polynomial function $q_{e}$ belonging to $L_{0}^{2}(K)$ such that

$$
\nabla q_{e}=f \quad \text { in each } K \in \mathcal{T}_{h}
$$

which leads to $\mathcal{M}_{K}^{u} \boldsymbol{f}=0$. Therefore, no enriching contribution comes from (26) but for the one related to $\boldsymbol{u}_{1}$.

Finally, based on the previous remark, and replacing (30) and (26)-(27) in (29), we arrive at the following final form of PGEM: Find $\left(\boldsymbol{u}_{1}, p_{0}\right) \in \mathbf{V}_{h} \times Q_{h}$ such that

$$
\begin{gathered}
\mathbf{A}_{s}\left(\left(\boldsymbol{u}_{1}-\sigma \mathcal{M}_{K}^{u}\left(\boldsymbol{u}_{1}\right), p_{0}\right),\left(\pi\left(\boldsymbol{v}_{1}\right), q_{0}\right)\right)+\sum_{K \in \mathcal{T}_{h}}\left(\mathcal{D}_{K}^{u}\left(\llbracket p_{0} \rrbracket\right), \sigma \pi\left(\boldsymbol{v}_{1}\right)\right)_{K} \\
-\sum_{F \in \mathcal{E}_{h}} \tau_{F}\left(\llbracket p_{0} \rrbracket, \llbracket q_{0} \rrbracket\right)_{F}=\mathbf{F}_{s}\left(\pi\left(\boldsymbol{v}_{1}\right), q_{0}\right),
\end{gathered}
$$

for all $\left(\boldsymbol{v}_{1}, q_{0}\right) \in \mathbf{V}_{h} \times Q_{h}$, which is precisely the method (13) since, as it will be shown in terms of the basis function in the next section, the following holds

$$
\pi_{K}\left(\boldsymbol{u}_{1}\right) \equiv\left(\mathcal{I}-\sigma \mathcal{M}_{K}^{u}\right)\left(\boldsymbol{u}_{1}\right) \quad \text { and } \quad \ell_{K}\left(\llbracket p_{0} \rrbracket\right) \equiv \mathcal{D}_{K}^{u}\left(\llbracket p_{0} \rrbracket\right) .
$$

Next, the symmetric method (16) follows by neglecting the non diagonal term $\left(\ell\left(\llbracket \hat{p}_{0} \rrbracket\right), \sigma \pi\left(\boldsymbol{v}_{1}\right)\right)_{\Omega}$ as this term does not undermine convergence estimates (see Section 3).

Remark. Following analogous steps and just replacing the forms $\mathbf{A}_{s}(.,$.$) and \mathbf{F}_{s}($.$) by \mathbf{A}(.,$. and $\mathbf{F}($.$) , and switching the velocity test space \pi\left(\mathbf{V}_{h}\right)$ to $\mathbf{V}_{h}$ in (18) we arrive at the following method: Find $\left(\hat{\boldsymbol{u}}_{1}, \hat{p}_{0}\right) \in \mathbf{V}_{h} \times Q_{h}$ such that

$$
\mathbf{A}\left(\left(\pi\left(\hat{\boldsymbol{u}}_{1}\right), \hat{p}_{0}\right),\left(\boldsymbol{v}_{1}, q_{0}\right)\right)+\left(\ell\left(\llbracket \hat{p}_{0} \rrbracket\right), \sigma \boldsymbol{v}_{1}\right)_{\Omega}+\sum_{F \in \mathcal{E}_{h}} \tau_{F}\left(\llbracket \hat{p}_{0} \rrbracket, \llbracket q_{0} \rrbracket\right)_{F}=\mathbf{F}\left(\boldsymbol{v}_{1}, q_{0}\right),
$$

for all $\left(\boldsymbol{v}_{1}, q_{0}\right) \in \mathbf{V}_{h} \times Q_{h}$, which is exactly the PGEM proposed in [7].

2.2. The local problems. This section is devoted to show the relationship between the local problems (26)-(27) and the Raviart-Thomas interpolation operator $\pi$ and the lifting operator $\ell$. First, we decompose $\boldsymbol{u}_{1}=\sum_{k=1}^{2} \sum_{i=1}^{3} u_{i}^{k} \psi_{i}^{k}$, where $u_{i}^{k}$ are the nodal values of $\boldsymbol{u}_{1}$ and $\boldsymbol{\psi}_{i}^{k}$, that denotes the (vector-valued) hat function. Then, we look for solutions of (26) and (27) in the form

$$
\left.\boldsymbol{u}_{e}^{D}\right|_{K}=\sum_{j=1}^{3} \frac{\alpha_{F_{j}} \int_{F_{j}} \llbracket p_{0} \rrbracket}{\sigma} \boldsymbol{\varphi}_{j} \quad \text { and }\left.\quad p_{e}^{D}\right|_{K}=\sum_{j=1}^{3} \frac{\alpha_{F_{j}} \int_{F_{j}} \llbracket p_{0} \rrbracket}{\sigma} \eta_{j},
$$


and

$$
\left.\boldsymbol{u}_{e}^{M}\right|_{K}=-\sigma \sum_{k=1}^{2} \sum_{i=1}^{3} u_{i}^{k} \bar{\varphi}_{i}^{k} \quad \text { and }\left.\quad p_{e}^{M}\right|_{K}=-\sigma \sum_{k=1}^{2} \sum_{i=1}^{3} u_{i}^{k} \bar{\eta}_{i}^{k}
$$

Here, indexes $j$ and $i$ are representing edges and local nodal numeration, respectively. Next, by replacing (33) and (34) in the local problems (27) and (26) respectively, and factoring out the coefficients it follows that the multi-scale basis functions $\left(\boldsymbol{\varphi}_{j}, \eta_{j}\right)$ and $\left(\overline{\boldsymbol{\varphi}}_{i}^{k}, \bar{\eta}_{i}^{k}\right)$ must satisfy the following well-posed local Darcy problems

$$
\begin{aligned}
& \sigma \boldsymbol{\varphi}_{j}+\nabla \eta_{j}=\mathbf{0}, \quad \nabla \cdot \boldsymbol{\varphi}_{j}=\frac{h_{F_{j}}}{|K|} \boldsymbol{n} \cdot \boldsymbol{n}_{F_{j}}^{K} \quad \text { in } K, \\
& \boldsymbol{\varphi}_{j} \cdot \boldsymbol{n}=\left\{\begin{array}{ll}
1 & \text { if } j=i, \\
0 & \text { otherwise }
\end{array} \quad \text { on each } F_{i} \subseteq \partial K,\right.
\end{aligned}
$$

and

$$
\begin{gathered}
\sigma \overline{\boldsymbol{\varphi}}_{i}^{k}+\nabla \bar{\eta}_{i}^{k}=\boldsymbol{\psi}_{i}^{k}, \quad \nabla \cdot \overline{\boldsymbol{\varphi}}_{i}^{k}=0 \quad \text { in } K, \\
\sigma \overline{\boldsymbol{\varphi}}_{i}^{k} \cdot \boldsymbol{n}=\boldsymbol{\psi}_{i}^{k} \cdot \boldsymbol{n}-\Pi_{F}\left(\boldsymbol{\psi}_{i}^{k} \cdot \boldsymbol{n}\right) \quad \text { on each } F \subseteq \partial K .
\end{gathered}
$$

The enrichment functions emanating from the problem (35)-(36) are nothing but the well known basis functions of the space $\mathrm{RT}_{0}(K)$, i.e, the lowest order Raviart-Thomas approximation of $H^{\text {div }}(K)$ defined by (6), and they are given by (cf. (10))

$$
\boldsymbol{\varphi}_{j}(\mathbf{x})= \pm \frac{h_{F_{j}}}{2|K|}\left(\mathbf{x}-\mathbf{x}_{F_{j}}\right) \quad \text { for } j=1,2,3 .
$$

Consequently,

$$
\eta_{j}(\mathbf{x})=\mp \frac{\sigma h_{F_{j}}}{2|K|}\left(\frac{|\mathbf{x}|^{2}}{2}-\mathbf{x} \cdot \mathbf{x}_{F_{j}}+C_{j}\right) \quad \text { for } j=1,2,3
$$

where the constant $C_{j}$ is set up so $\int_{K} \eta_{j}=0$. The solution of (37)-(38) can also be analytically computed, providing

$$
\sigma \overline{\boldsymbol{\varphi}}_{i}^{k}=\boldsymbol{\psi}_{i}^{k}-\sum_{j=1}^{3} \Pi_{F}\left(\boldsymbol{\psi}_{i}^{k} \cdot \boldsymbol{n}\right) \boldsymbol{\varphi}_{j}=\boldsymbol{\psi}_{i}^{k}-\pi_{K}\left(\boldsymbol{\psi}_{i}^{k}\right)
$$

where $\pi_{K}$ has been defined in (9). A similar local problem has been used in [15] to obtain multiscale basis functions for the Darcy problem with oscillating coefficients (see also [1] for the extension to porous media with stochastic coefficients). 
2.3. The local mass conservation feature: a general strategy. Computing numerical solutions through PGEM (13) and the symmetric method (16) does not assure local mass conservative velocity field if only linear part of solution $\boldsymbol{u}_{1}$ (or $\hat{\boldsymbol{u}}_{1}$ ) is considered. Regarding the first method, the required feature is achieved by locally updating $\boldsymbol{u}_{1}$ with $\boldsymbol{u}_{e}^{D}$ given by (27). In fact, since discontinuous pressure interpolations are used it emerges from (13) that (see [7] for a related idea)

$$
\int_{K} \nabla \cdot\left(\boldsymbol{u}_{1}+\boldsymbol{u}_{e}^{D}\right)=\int_{K} g, \quad \text { for all } K \in \mathcal{T}_{h}
$$

Remark. Summing up, we see that in order to obtain a stable pair of interpolation spaces with discrete velocity field locally mass-conservative, it is fundamental to enrich the linear part of the discrete velocity $\boldsymbol{u}_{1}$ with an element of the Raviart-Thomas' space $\mathbf{V}_{R T_{0}}$, namely, the multi-scale function $\boldsymbol{u}_{e}^{D}$ computed from (33). We stress the fact that the computation of (33) follows directly from the discrete solution, without the need of any extra local computation. Moreover, the exact velocity $\boldsymbol{u}$ is approximated in each $K \in \mathcal{T}_{h}$ by

$$
\begin{aligned}
\boldsymbol{u}_{h} & =\boldsymbol{u}_{1}+\boldsymbol{u}_{e}^{M}+\boldsymbol{u}_{e}^{D} \\
& =\sum_{k=1}^{2} \sum_{i=1}^{3} u_{i}^{k} \boldsymbol{\psi}_{i}^{k}-\sigma \sum_{k=1}^{2} \sum_{i=1}^{3} u_{i}^{k} \overline{\boldsymbol{\varphi}}_{i}^{k}+\sum_{l=1}^{3} \frac{\alpha_{F_{l}}}{\sigma} \int_{F_{l}} \llbracket p_{0} \rrbracket \boldsymbol{\varphi}_{l} \\
& =\sum_{l=1}^{3}\left(\Pi_{F_{l}}\left(\boldsymbol{u}_{1} \cdot \boldsymbol{n}\right)+\tau_{F_{l}} \Pi_{F_{l}}\left(\llbracket p_{0} \rrbracket\right)\right) \boldsymbol{\varphi}_{l} \\
& =\pi_{K}\left(\boldsymbol{u}_{1}\right)+\ell_{K}\left(\llbracket p_{0} \rrbracket\right)
\end{aligned}
$$

so, as expected, the continuity of the normal velocity component through the internal edges is assured, but not the tangential one.

Now, it turns out that such local mass recovering is not only restricted to methods arising exactly from the enhancing approach. For example, stabilized methods based on pressure jumps as the one presented in [14], or the symmetric method (16) are elegible to recover the local mass conservation feature adding $\ell_{K}\left(\llbracket p_{0} \rrbracket\right)$ to the computed velocity field, where $p_{0}$ is the constant pressure solution. We illustrate this fact for the symmetric formulation (16). Choosing $\boldsymbol{v}_{1}=\mathbf{0}$ in (16) and $q_{0}=1$ in $K$ and $-|K| /\left|K^{\prime}\right|$ in $K^{\prime}$ (where $K \cap K^{\prime}=F \in \mathcal{E}_{h}$ ), we obtain after integration by parts and the definition of $\ell_{K}$ that

$$
\begin{gathered}
\int_{K} \nabla \cdot\left(\hat{\boldsymbol{u}}_{1}+\ell_{K}\left(\llbracket \hat{p}_{0} \rrbracket\right)\right)-\frac{|K|}{\left|K^{\prime}\right|} \int_{K^{\prime}} \nabla \cdot\left(\hat{\boldsymbol{u}}_{1}+\ell_{K}\left(\llbracket \hat{p}_{0} \rrbracket\right)\right)= \\
\int_{K} \nabla \cdot \hat{\boldsymbol{u}}_{1}-\frac{|K|}{\left|K^{\prime}\right|} \int_{K^{\prime}} \nabla \cdot \hat{\boldsymbol{u}}_{1}+\sum_{F \subseteq \partial K \cup \partial K^{\prime}} \tau_{F}\left(\llbracket \hat{p}_{0} \rrbracket, \llbracket q_{0} \rrbracket\right)_{F}=\int_{K} g-\frac{|K|}{\left|K^{\prime}\right|} \int_{K^{\prime}} g,
\end{gathered}
$$


and then, following closely the arguments given in [7], we obtain that the value $\int_{K} \nabla \cdot\left(\hat{\boldsymbol{u}}_{1}-\right.$ $\left.\ell_{K}\left(\llbracket \hat{p}_{0} \rrbracket\right)\right)-g$ vanishes in each $K$, and hence it must vanish on each element. It is important to emphasize once more that $\ell\left(\llbracket \hat{p}_{0} \rrbracket\right)$ does not perturb too much the solution in the sense that the order of error estimates is still preserved (see Theorems 8 and 9 for details).

\section{ERROR ANALYSIS}

In the sequel $C$ denotes a generic positive constant, independent of $h$ or $\sigma$, with values that may vary in each occurrence. Before performing an error analysis of (13) and (16), we need to consider interpolation inequalities to approximate variables.

3.1. Interpolation, stability and consistency results. We start by presenting the Clément interpolation operator (cf. $[16,22,17]) \mathcal{C}_{h}: H^{1}(\Omega) \rightarrow V_{h}$ (with the obvious extension to vector-valued functions), satisfying, for all $K \in \mathcal{T}_{h}$ and all $F \in \mathcal{E}_{h}$,

$$
\begin{aligned}
\left\|v-\mathcal{C}_{h}(v)\right\|_{m, K} \leq C h_{K}^{t-m}|v|_{t, \omega_{K}} \quad \forall v \in H^{t}\left(\omega_{K}\right), \\
\left\|v-\mathcal{C}_{h}(v)\right\|_{0, F} \leq C h_{F}^{t-\frac{1}{2}}|v|_{t, \omega_{F}} \quad \forall v \in H^{t}\left(\omega_{F}\right),
\end{aligned}
$$

for $t=1,2, m=0,1$, where $\omega_{K}=\left\{K^{\prime} \in \mathcal{T}_{h}: K \cap K^{\prime} \neq \emptyset\right\}$ and $\omega_{F}=\left\{K \in \mathcal{T}_{h}: K \cap F \neq \emptyset\right\}$.

Now, in order to take into account the approximation of the pressure and the consistency error, we consider the $L^{2}(\Omega)$ projection onto $Q_{h}$ which is denoted by $\Pi_{h}: L^{2}(\Omega) \rightarrow Q_{h}$. This projection satisfies (cf. [17])

$$
\left\|q-\Pi_{h}(q)\right\|_{m, \Omega} \leq C h^{1-m}|q|_{1, \Omega} \quad \forall q \in H^{1}(\Omega),
$$

for $m=0,1$. Moreover, using the result above and the following local trace inequality: given $K \in \mathcal{T}_{h}, F \subseteq \partial K$, there exists $C$ such that for all $v \in H^{1}(K)$

$$
\|v\|_{0, F}^{2} \leq C\left(\frac{1}{h_{K}}\|v\|_{0, K}^{2}+h_{K}|v|_{1, K}^{2}\right)
$$

we obtain

$$
\left[\sum_{F \in \mathcal{E}_{h}} h_{F}\left\|\llbracket q-\Pi_{h}(q) \rrbracket\right\|_{0, F}^{2}\right]^{1 / 2} \leq C h|q|_{1, \Omega} .
$$

Moreover, we will systematically use the Raviart-Thomas interpolation operator $\pi$ defined through (9) as

$$
\pi(\boldsymbol{v})=\sum_{F \in \mathcal{E}_{h}} \Pi_{F}\left(\left.\boldsymbol{v} \cdot \boldsymbol{n}\right|_{F}\right) \boldsymbol{\varphi}_{F},
$$

satisfying (see [17] or Corollary 6 for an alternative proof)

$$
\|\boldsymbol{v}-\pi(\boldsymbol{v})\|_{0, \Omega} \leq C h|\boldsymbol{v}|_{1, \Omega},
$$


for all $\boldsymbol{v} \in H^{1}(\Omega)^{2}$ and

$$
\|\nabla \cdot \boldsymbol{v}-\nabla \cdot \pi(\boldsymbol{v})\|_{0, \Omega} \leq C h|\nabla \cdot \boldsymbol{v}|_{1, \Omega}
$$

for all $\boldsymbol{v} \in H^{1}(\Omega)^{2}$ such that $\nabla \cdot \boldsymbol{v} \in H^{1}(\Omega)$.

Now, we define the following mesh dependent norm

$$
\|(\boldsymbol{v}, q)\|_{h}:=\left(\sum_{K \in \mathcal{T}_{h}} \sigma\|\boldsymbol{v}\|_{0, K}^{2}+\sum_{F \in \mathcal{E}_{h}} \tau_{F}\|\llbracket q \rrbracket\|_{0, F}^{2}\right)^{1 / 2},
$$

and we present an interpolation result in this norm.

Lemma 1. Let us suppose that $(\boldsymbol{v}, q) \in H^{1}(\Omega)^{2} \times H^{1}(\Omega)$. Then, there exists $C$ such that

$$
\left\|\left(\boldsymbol{v}-\pi\left(\mathcal{C}_{h}(\boldsymbol{v})\right), q-\Pi_{h}(q)\right)\right\|_{h} \leq C h\left(\sqrt{\sigma}|\boldsymbol{v}|_{1, \Omega}+\frac{1}{\sqrt{\sigma}}|q|_{1, \Omega}\right) .
$$

Proof. From the definition of the norm, (42) and (46) there follows that

$$
\begin{aligned}
\|\left(\boldsymbol{v}-\pi\left(\mathcal{C}_{h}(\boldsymbol{v})\right),\right. & \left.q-\Pi_{h}(q)\right)\left\|_{h}^{2}=\sigma\right\| \boldsymbol{v}-\pi\left(\mathcal{C}_{h}(\boldsymbol{v})\right)\left\|_{0, \Omega}^{2}+\sum_{F \in \mathcal{E}_{h}} \tau_{F}\right\| \llbracket q-\Pi_{h}(q) \rrbracket \|_{0, F}^{2} \\
\leq & 2 \sigma\left(\|\boldsymbol{v}-\pi(\boldsymbol{v})\|_{0, \Omega}^{2}+\left\|\pi\left(\boldsymbol{v}-\mathcal{C}_{h}(\boldsymbol{v})\right)\right\|_{0, \Omega}^{2}\right)+\sum_{F \in \mathcal{E}_{h}} \tau_{F}\left\|\llbracket q-\Pi_{h}(q) \rrbracket\right\|_{0, F}^{2} \\
& \leq C\left(\sigma h^{2}|\boldsymbol{v}|_{1, \Omega}^{2}+\frac{h^{2}}{\sigma}|q|_{1, \Omega}^{2}\right)+2 \sigma\left\|\pi\left(\boldsymbol{v}-\mathcal{C}_{h}(\boldsymbol{v})\right)\right\|_{0, \Omega}^{2} .
\end{aligned}
$$

Next, from its definition it is easy to prove that the Raviart-Thomas operator $\pi$ satisfies (see [9] for a related result and Lemma 5 for an alternative proof)

$$
\|\pi(\boldsymbol{v})\|_{0, \Omega} \leq C\left(\|\boldsymbol{v}\|_{0, \Omega}+h|\boldsymbol{v}|_{1, \Omega}\right)
$$

for all $\boldsymbol{v} \in H^{1}(\Omega)^{2}$, and then the result follows applying (52) and (42) in (51).

Before heading to stability, an auxiliary result is stated next.

Lemma 2. Let $\pi$ be the Raviart-Thomas interpolator, then there exists a positive constant $C_{1}$ such that, for all $\boldsymbol{v}_{1} \in \mathbf{V}_{h}$ and $q_{0} \in Q_{h}$, it holds

$$
\left(\ell\left(\llbracket q_{0} \rrbracket\right), \sigma \pi\left(\boldsymbol{v}_{1}\right)\right)_{\Omega} \leq C_{1}\left\{\sum_{F \in \mathcal{E}_{h}} \tau_{F}\left\|\llbracket q_{0} \rrbracket\right\|_{0, F}^{2}\right\}^{\frac{1}{2}} \sqrt{\sigma \alpha} h\left\|\pi\left(\boldsymbol{v}_{1}\right)\right\|_{0, \Omega}
$$

where $\alpha:=\max \left\{\alpha_{F}: F \in \mathcal{E}_{h}\right\}$. 
Proof. In order to prove (53) we first note that $\ell\left(\llbracket q_{0} \rrbracket\right) \in \mathbf{V}_{R T_{0}}$. Hence, using successively the Cauchy-Schwarz inequality, (11) and $\left\|\boldsymbol{\varphi}_{F}\right\|_{0, K} \leq C_{1} h_{F}$ we get

$$
\begin{aligned}
\left(\ell\left(\llbracket q_{0} \rrbracket\right), \sigma \pi\left(\boldsymbol{v}_{1}\right)\right)_{\Omega} & \leq \sum_{K \in \mathcal{T}_{h}}\left\|\ell_{K}\left(\llbracket q_{0} \rrbracket\right)\right\|_{0, K} \sigma\left\|\pi\left(\boldsymbol{v}_{1}\right)\right\|_{0, K} \\
& \leq \sum_{K \in \mathcal{T}_{h}} \sum_{F \subseteq \partial K} \frac{\alpha_{F}}{\sigma} \int_{F} \mid \llbracket q_{0} \rrbracket\left\|\boldsymbol{\varphi}_{F}\right\|_{0, K} \sigma\left\|\pi\left(\boldsymbol{v}_{1}\right)\right\|_{0, K} \\
& \leq C_{1} \sum_{K \in \mathcal{T}_{h}} \sum_{F \subseteq \partial K} \tau_{F} h_{F}^{\frac{1}{2}}\left\|\llbracket q_{0} \rrbracket\right\|_{0, F} \sigma\left\|\pi\left(\boldsymbol{v}_{1}\right)\right\|_{0, K} \\
& \leq C_{1}\left\{\sum_{F \in \mathcal{E}_{h}} \tau_{F}\left\|\llbracket q_{0} \rrbracket\right\|_{0, F}^{2}\right\}^{\frac{1}{2}} h \sqrt{\sigma \alpha}\left\|\pi\left(\boldsymbol{v}_{1}\right)\right\|_{0, \Omega}
\end{aligned}
$$

and the result follows.

We are ready to prove PGEM are well-posed.

Lemma 3. The bilinear forms $\mathbf{B}_{s}(.,$.$) defined in (17) satisfies$

$$
\mathbf{B}_{s}\left(\left(\boldsymbol{v}_{1}, q_{0}\right),\left(\boldsymbol{v}_{1},-q_{0}\right)\right)=\left\|\left(\pi\left(\boldsymbol{v}_{1}\right), q_{0}\right)\right\|_{h}^{2} \quad \forall\left(\boldsymbol{v}_{1}, q_{0}\right) \in \mathbf{V}_{h} \times Q_{h}
$$

Moreover, assuming $\alpha \leq \frac{1}{C_{1}^{2} h^{2}}$ where $C_{1}$ is the positive constant from Lemma 2 , the bilinear form $\mathbf{B}(.,$.$) defined in (14) satisfies,$

$$
\mathbf{B}\left(\left(\boldsymbol{v}_{1}, q_{0}\right),\left(\boldsymbol{v}_{1},-q_{0}\right)\right) \geq \frac{1}{2}\left\|\left(\pi\left(\boldsymbol{v}_{1}\right), q_{0}\right)\right\|_{h}^{2} \quad \forall\left(\boldsymbol{v}_{1}, q_{0}\right) \in \mathbf{V}_{h} \times Q_{h} .
$$

Hence, the problems (13) and (16) are well-posed.

Proof. The first equality follows directly from the definition of the bilinear form $\mathbf{B}_{s}(.,$.$) . For$ the second one, we recall that

$$
\mathbf{B}\left(\left(\boldsymbol{v}_{1}, q_{0}\right),\left(\boldsymbol{v}_{1},-q_{0}\right)\right)=\sigma\left\|\pi\left(\boldsymbol{v}_{1}\right)\right\|_{\Omega}^{2}+\left(\ell\left(\llbracket q_{0} \rrbracket\right), \sigma \pi\left(\boldsymbol{v}_{1}\right)\right)_{\Omega}+\sum_{F \in \mathcal{E}_{h}} \tau_{F}\left\|\llbracket q_{0} \rrbracket\right\|_{0, F}^{2} .
$$

As for the second term, we use Lemma 2 to obtain

$$
\begin{aligned}
\left(\ell\left(\llbracket q_{0} \rrbracket\right), \sigma \pi\left(\boldsymbol{v}_{1}\right)\right)_{\Omega} & \leq C_{1}\left\{\sum_{F \in \mathcal{E}_{h}} \tau_{F}\left\|\llbracket q_{0} \rrbracket\right\|_{0, F}^{2}\right\}^{\frac{1}{2}} h \sqrt{\sigma \alpha}\left\|\pi\left(\boldsymbol{v}_{1}\right)\right\|_{0, \Omega} \\
& \leq \sum_{F \in \mathcal{E}_{h}} \frac{1}{2} C_{1}^{2} \alpha h^{2} \tau_{F}\left\|\llbracket q_{0} \rrbracket\right\|_{0, F}^{2}+\frac{1}{2} \sigma\left\|\pi\left(\boldsymbol{v}_{1}\right)\right\|_{0, \Omega}^{2}
\end{aligned}
$$


Hence by successively applying inequality above into (54) and assuming $\alpha \leq \frac{1}{C_{1}^{2} h^{2}}$ it holds

$$
\begin{aligned}
\mathbf{B}\left(\left(\boldsymbol{v}_{1}, q_{0}\right),\left(\boldsymbol{v}_{1},-q_{0}\right)\right) & \geq \frac{\sigma}{2}\left\|\pi\left(\boldsymbol{v}_{1}\right)\right\|_{0, \Omega}^{2}+\sum_{F \in \mathcal{E}_{h}}\left(1-\frac{C_{1}^{2} \alpha h^{2}}{2}\right) \tau_{F}\left\|\llbracket q_{0} \rrbracket\right\|_{0, F}^{2} \\
& \geq \frac{1}{2}\left\|\left(\pi\left(\boldsymbol{v}_{1}\right), q_{0}\right)\right\|_{h}^{2} .
\end{aligned}
$$

The continuity of bilinear forms is straightforward and thus the well-posedness of (13) and (16) stems from the Necas Theorem [17].

Remark. We remark that the metric $\left\|\left(\pi\left(\boldsymbol{v}_{1}\right), q_{0}\right)\right\|_{h}$ defines a norm in the space $\mathbf{V}_{h} \times Q_{h}$ since the Raviart-Thomas interpolation operator is injective when restricted to $\mathbf{V}_{h}$. On the other hand, we remark that the hypothesis on $\alpha$ is not really restrictive, since it only applies for coarse meshes. For a sufficiently refined mesh the choice for $\alpha$ is essentially unlimited.

Neither of the methods proposed in the previous section are formally consistent as points out the next result.

Lemma 4. Let $(\boldsymbol{u}, p) \in H_{0}^{\text {div }}(\Omega) \times\left[H^{1}(\Omega) \cap L_{0}^{2}(\Omega)\right]$ be the weak solution of $(3),\left(\boldsymbol{u}_{1}, p_{0}\right)$ the solution of $(13)$ and $\left(\hat{\boldsymbol{u}}_{1}, \hat{p}_{0}\right)$ the solution of (16), respectively. Then,

$$
\begin{gathered}
\mathbf{B}\left(\left(\boldsymbol{u}-\boldsymbol{u}_{1}, p-p_{0}\right),\left(\boldsymbol{v}_{1}, q_{0}\right)\right)=-\sum_{K \in \mathcal{T}_{h}}\left(\sigma \mathcal{M}_{K}^{u}(\boldsymbol{u}), \sigma \pi_{K}\left(\boldsymbol{v}_{1}\right)\right)_{K}, \\
\mathbf{B}_{s}\left(\left(\boldsymbol{u}-\hat{\boldsymbol{u}}_{1}, p-\hat{p}_{0}\right),\left(\boldsymbol{v}_{1}, q_{0}\right)\right)=-\sum_{K \in \mathcal{T}_{h}}\left(\sigma \mathcal{M}_{K}^{u}(\boldsymbol{u}), \sigma \pi_{K}\left(\boldsymbol{v}_{1}\right)\right)_{K},
\end{gathered}
$$

for all $\left(\boldsymbol{v}_{1}, q_{0}\right) \in \mathbf{V}_{h} \times Q_{h}$.

Proof. The result follows from the definition of $\mathbf{B}(.,$.$) and \mathbf{B}_{s}(.,$.$) , and noting that \llbracket p \rrbracket=0$ a.e. across all the internal edges

3.2. Error estimates for the symmetric formulation. We begin this section by proving the following technical result concerning the operator $\mathcal{M}_{K}^{u}$.

Lemma 5. Let $\boldsymbol{v} \in H^{1}(K)^{2}$. Then, there exists a constant $C$ such that

$$
\left\|\mathcal{M}_{K}^{u}(\boldsymbol{v})\right\|_{0, K} \leq C \sigma^{-1} h_{K}|\boldsymbol{v}|_{1, K}
$$

Proof. Let $\boldsymbol{v} \in H^{1}(K)^{2}$, and $\boldsymbol{w}:=\mathcal{M}_{K}^{u}(\boldsymbol{v})$. Then, from the definition of $\mathcal{M}_{K}^{u}$ (cf. (24)), $\boldsymbol{w}$ satisfies

$$
\begin{gathered}
\sigma \boldsymbol{w}+\nabla \xi=\boldsymbol{v}, \quad \sigma \nabla \cdot \boldsymbol{w}=\nabla \cdot \boldsymbol{v}-\Pi_{K}(\nabla \cdot \boldsymbol{v}) \quad \text { in } K, \\
\sigma \boldsymbol{w} \cdot \boldsymbol{n}=\boldsymbol{v} \cdot \boldsymbol{n}-\Pi_{F}(\boldsymbol{v} \cdot \boldsymbol{n}) \quad \text { on each } F \subseteq \partial K,
\end{gathered}
$$


where $\xi \in L_{0}^{2}(K)$. Multiplying the first equation by $\boldsymbol{w}$ and integrating it by parts we arrive at

$$
\begin{aligned}
\sigma\|\boldsymbol{w}\|_{0, K}^{2}= & (\boldsymbol{v}, \boldsymbol{w})_{K}+\frac{1}{\sigma}\left(\xi, \nabla \cdot \boldsymbol{v}-\Pi_{K}(\nabla \cdot \boldsymbol{v})\right)_{K}-\frac{1}{\sigma} \sum_{F \subseteq \partial K}\left(\xi, \boldsymbol{v} \cdot \boldsymbol{n}-\Pi_{F}(\boldsymbol{v} \cdot \boldsymbol{n})\right)_{F} \\
= & (\boldsymbol{v}, \boldsymbol{w})_{K}+\frac{1}{\sigma}\left(\xi-\Pi_{K}(\xi), \nabla \cdot \boldsymbol{v}-\Pi_{K}(\nabla \cdot \boldsymbol{v})\right)_{K} \\
& -\frac{1}{\sigma} \sum_{F \subseteq \partial K}\left(\xi-\Pi_{F}(\xi), \boldsymbol{v} \cdot \boldsymbol{n}-\Pi_{F}(\boldsymbol{v} \cdot \boldsymbol{n})\right)_{F} \\
\leq & \|\boldsymbol{v}\|_{0, K}\|\boldsymbol{w}\|_{0, K}+\frac{1}{\sigma}\left\|\xi-\Pi_{K}(\xi)\right\|_{0, K}\left\|\nabla \cdot \boldsymbol{v}-\Pi_{K}(\nabla \cdot \boldsymbol{v})\right\|_{0, K} \\
& +\frac{1}{\sigma} \sum_{F \subseteq \partial K}\left\|\xi-\Pi_{F}(\xi)\right\|_{0, F}\left\|\boldsymbol{v} \cdot \boldsymbol{n}-\Pi_{F}(\boldsymbol{v} \cdot \boldsymbol{n})\right\|_{0, F} \cdot
\end{aligned}
$$

Next, from (55) it holds $\|\nabla \xi\|_{0, K} \leq\|\boldsymbol{v}\|_{0, K}+\sigma\|\boldsymbol{w}\|_{0, K}$ and hence, using the local trace result (45), the approximation property of the projection operators $\Pi_{F}$ and $\Pi_{K}$, and the inequality above to obtain

$$
\begin{aligned}
\sigma\|\boldsymbol{w}\|_{0, K}^{2} & \leq\|\boldsymbol{v}\|_{0, K}\|\boldsymbol{w}\|_{0, K}+\frac{C}{\sigma} h_{K}|\xi|_{1, K}|\boldsymbol{v}|_{1, K} \\
& \leq\|\boldsymbol{v}\|_{0, K}\|\boldsymbol{w}\|_{0, K}+\frac{C h_{K}}{\sigma}\left(\|\boldsymbol{v}\|_{0, K}+\sigma\|\boldsymbol{w}\|_{0, K}\right)|\boldsymbol{v}|_{1, K} \\
& \leq C \sigma^{-1}\left(\|\boldsymbol{v}\|_{0, K}^{2}+h_{K}^{2}|\boldsymbol{v}|_{1, K}^{2}\right)+\frac{\sigma}{2}\|\boldsymbol{w}\|_{0, K}^{2}
\end{aligned}
$$

and then we have proved that

$$
\left\|\mathcal{M}_{K}^{u}(\boldsymbol{v})\right\|_{0, K} \leq C \sigma^{-1}\left(\|\boldsymbol{v}\|_{0, K}+h_{K}|\boldsymbol{v}|_{1, K}\right)
$$

Finally, let us denote $\boldsymbol{v}_{0}=\Pi_{K}(\boldsymbol{v})$. Since $\boldsymbol{v}_{0}$ is a constant in each element, there holds that $\mathcal{M}_{K}^{u}\left(\boldsymbol{v}_{0}\right)=\mathbf{0}$ and then, from (58) it follows that

$$
\left\|\mathcal{M}_{K}^{u}(\boldsymbol{v})\right\|_{0, K}=\left\|\mathcal{M}_{K}^{u}\left(\boldsymbol{v}-\boldsymbol{v}_{0}\right)\right\|_{0, K} \leq C \sigma^{-1}\left(\left\|\boldsymbol{v}-\boldsymbol{v}_{0}\right\|_{0, K}+h_{K}|\boldsymbol{v}|_{1, K}\right)
$$

and the result follows using the approximation properties of the projection.

The previous lemma results in an alternative proof of the following classical interpolation error estimate:

Corollary 6. There exists $C$ such that

$$
\left\|\boldsymbol{v}-\pi_{K}(\boldsymbol{v})\right\|_{0, K} \leq C h_{K}|\boldsymbol{v}|_{1, K}
$$

for all $\boldsymbol{v} \in H^{1}(K)^{2}$. 
Proof. The result follows from the previous lemma and the fact that $\boldsymbol{v}-\pi_{K}(\boldsymbol{v})=\sigma \mathcal{M}_{K}^{u}(\boldsymbol{v})$.

Theorem 7. Let $(\boldsymbol{u}, p) \in H_{0}^{d i v}(\Omega) \cap H^{2}(\Omega)^{2} \times H^{1}(\Omega) \cap L_{0}^{2}(\Omega)$ be the solution of (2), and $\left(\hat{\boldsymbol{u}}_{1}, \hat{p}_{0}\right) \in \mathbf{V}_{h} \times Q_{h}$ the solution of method (16). Then, defining $\hat{\boldsymbol{u}}_{h}:=\pi\left(\hat{\boldsymbol{u}}_{1}\right)$, the following error estimate holds

$$
\left\|\left(\boldsymbol{u}-\hat{\boldsymbol{u}}_{h}, p-\hat{p}_{0}\right)\right\|_{h} \leq C h\left(\sqrt{\sigma}\|\boldsymbol{u}\|_{2, \Omega}+\frac{1}{\sqrt{\sigma}}|p|_{1, \Omega}\right)
$$

Proof. Let $\left(\boldsymbol{v}_{1}, q_{0}\right)=\left(\mathcal{C}_{h}(\boldsymbol{u}), \Pi_{h}(p)\right)$. From the triangle inequality we have

$$
\left\|\left(\boldsymbol{u}-\pi\left(\hat{\boldsymbol{u}}_{1}\right), p-\hat{p}_{0}\right)\right\|_{h} \leq\left\|\left(\boldsymbol{u}-\pi\left(\boldsymbol{v}_{1}\right), p-q_{0}\right)\right\|_{h}+\left\|\left(\pi\left(\boldsymbol{v}_{1}-\hat{\boldsymbol{u}}_{1}\right), q_{0}-\hat{p}_{0}\right)\right\|_{h}
$$

The first term is easily estimated using Lemma 1. Next, let us estimate the second term on the right hand side. For that, we use the coercivity of $\mathbf{B}_{s}(.,$.$) (cf. Lemma 3) and the$ consistency result (cf. Lemma 4) to obtain

$$
\begin{aligned}
\left\|\left(\pi\left(\boldsymbol{v}_{1}-\hat{\boldsymbol{u}}_{1}\right), q_{0}-\hat{p}_{0}\right)\right\|_{h}^{2}=\mathbf{B}_{s}\left(\left(\boldsymbol{v}_{1}-\hat{\boldsymbol{u}}_{1}, q_{0}-\hat{p}_{0}\right),\left(\boldsymbol{v}_{1}-\hat{\boldsymbol{u}}_{1}, \hat{p}_{0}-q_{0}\right)\right) \\
=-\mathbf{B}_{s}\left(\left(\boldsymbol{u}-\boldsymbol{v}_{1}, p-q_{0}\right),\left(\boldsymbol{v}_{1}-\hat{\boldsymbol{u}}_{1}, \hat{p}_{0}-q_{0}\right)\right)-\sigma^{2} \sum_{K \in \mathcal{T}_{h}}\left(\mathcal{M}_{K}^{u}(\boldsymbol{u}), \pi_{K}\left(\boldsymbol{v}_{1}-\hat{\boldsymbol{u}}_{1}\right)\right)_{K} \\
=-\sigma\left(\pi\left(\boldsymbol{u}-\boldsymbol{v}_{1}\right), \pi\left(\boldsymbol{v}_{1}-\hat{\boldsymbol{u}}_{1}\right)\right)_{\Omega}+\left(p-q_{0}, \nabla \cdot\left(\pi\left(\boldsymbol{v}_{1}-\hat{\boldsymbol{u}}_{1}\right)\right)\right)_{\Omega}+\left(\hat{p}_{0}-q_{0}, \nabla \cdot\left(\pi\left(\boldsymbol{u}-\boldsymbol{v}_{1}\right)\right)\right)_{\Omega} \\
\quad+\sum_{F \in \mathcal{E}_{h}} \tau_{F}\left(\llbracket p-q_{0} \rrbracket, \llbracket \hat{p}_{0}-q_{0} \rrbracket\right)_{F}-\sum_{K \in \mathcal{T}_{h}} \sigma^{2}\left(\mathcal{M}_{K}^{u}(\boldsymbol{u}), \pi_{K}\left(\boldsymbol{v}_{1}-\hat{\boldsymbol{u}}_{1}\right)\right)_{K} \cdot
\end{aligned}
$$

Next, from the properties of the projection operator $\Pi_{h}$ it holds

$$
\left(p-q_{0}, \nabla \cdot\left(\pi\left(\boldsymbol{v}_{1}-\hat{\boldsymbol{u}}_{1}\right)\right)\right)_{\Omega}=0
$$


and then, integrating by parts and using the Cauchy-Schwarz's inequality we arrive at

$$
\begin{aligned}
&\left\|\left(\pi\left(\boldsymbol{v}_{1}-\hat{\boldsymbol{u}}_{1}\right), q_{0}-\hat{p}_{0}\right)\right\|_{h}^{2} \leq \sigma\left\|\pi\left(\boldsymbol{u}-\boldsymbol{v}_{1}\right)\right\|_{0, \Omega}\left\|\pi\left(\boldsymbol{v}_{1}-\hat{\boldsymbol{u}}_{1}\right)\right\|_{0, \Omega}+\sum_{F \in \mathcal{E}_{h}}\left(\llbracket \hat{p}_{0}-q_{0} \rrbracket,\left(\boldsymbol{u}-\boldsymbol{v}_{1}\right) \cdot \boldsymbol{n}\right)_{F} \\
& \quad+\sum_{F \in \mathcal{E}_{h}} \tau_{F}\left\|\llbracket p-q_{0} \rrbracket\right\|_{0, F}\left\|\llbracket \hat{p}_{0}-q_{0} \rrbracket\right\|_{0, F}+\sigma^{2} \sum_{K \in \mathcal{T}_{h}}\left(\mathcal{M}_{K}^{u}(\boldsymbol{u}), \pi_{K}\left(\boldsymbol{v}_{1}-\hat{\boldsymbol{u}}_{1}\right)\right)_{K} \\
& \leq \sigma\left\|\pi\left(\boldsymbol{u}-\boldsymbol{v}_{1}\right)\right\|_{0, \Omega}\left\|\pi\left(\boldsymbol{v}_{1}-\hat{\boldsymbol{u}}_{1}\right)\right\|_{0, \Omega}+C \sum_{F \in \mathcal{E}_{h}}\left\|\llbracket \hat{p}_{0}-q_{0} \rrbracket\right\|_{0, F} h_{F}^{\frac{3}{2}}|\boldsymbol{u}|_{2, \omega_{F}} \\
&+\sum_{F \in \mathcal{E}_{h}} \tau_{F}\left\|\llbracket p-q_{0} \rrbracket\right\|_{0, F}\left\|\llbracket \hat{p}_{0}-q_{0} \rrbracket\right\|_{0, F}+\sigma^{2} \sum_{K \in \mathcal{T}_{h}}\left(\mathcal{M}_{K}^{u}(\boldsymbol{u}), \pi_{K}\left(\boldsymbol{v}_{1}-\hat{\boldsymbol{u}}_{1}\right)\right)_{K} \\
& \leq \sigma\left\|\pi\left(\boldsymbol{u}-\boldsymbol{v}_{1}\right)\right\|_{0, \Omega}\left\|\pi\left(\boldsymbol{v}_{1}-\hat{\boldsymbol{u}}_{1}\right)\right\|_{0, \Omega}+C \sum_{F \in \mathcal{E}_{h}} \sigma^{\frac{1}{2}} \tau_{F}^{\frac{1}{2}}\left\|\llbracket \hat{p}_{0}-q_{0} \rrbracket\right\|_{0, F} h_{F}|\boldsymbol{u}|_{2, \omega_{F}} \\
&+\sum_{F \in \mathcal{E}_{h}} \tau_{F}\left\|\llbracket p-q_{0} \rrbracket\right\|_{0, F}\left\|\llbracket \hat{p}_{0}-q_{0} \rrbracket\right\|_{0, F}+\sigma^{2} \sum_{K \in \mathcal{T}_{h}}\left(\mathcal{M}_{K}^{u}(\boldsymbol{u}), \pi_{K}\left(\boldsymbol{v}_{1}-\hat{\boldsymbol{u}}_{1}\right)\right)_{K} \\
& \leq C\left\{\sigma\left\|\pi\left(\boldsymbol{u}-\boldsymbol{v}_{1}\right)\right\|_{0, \Omega}^{2}+\sigma h^{2}|\boldsymbol{u}|_{2, \Omega}^{2}+\sum_{F \in \mathcal{E}_{h}} \tau_{F}\left\|\llbracket p-q_{0} \rrbracket\right\|_{0, F}^{2}\right\}^{\frac{1}{2}}\left\|\left(\pi\left(\boldsymbol{v}_{1}-\hat{\boldsymbol{u}}_{1}\right), q_{0}-\hat{p}_{0}\right)\right\|_{h} \\
&+\sigma^{2} \sum_{K \in \mathcal{T}_{h}}\left(\mathcal{M}_{K}^{u}(\boldsymbol{u}), \pi_{K}\left(\boldsymbol{v}_{1}-\hat{\boldsymbol{u}}_{1}\right)\right)_{K} .
\end{aligned}
$$

The last term on the right hand side is estimated next. Using Lemma 5 we may bound the consistency term as follows

$$
\begin{aligned}
\sum_{K \in \mathcal{T}_{h}}\left(\mathcal{M}_{K}^{u}(\boldsymbol{u}), \pi_{K}\left(\boldsymbol{v}_{1}-\hat{\boldsymbol{u}}_{1}\right)\right)_{K} & \leq \sum_{K \in \mathcal{T}_{h}}\left\|\mathcal{M}_{K}^{u}(\boldsymbol{u})\right\|_{0, K}\left\|\pi_{K}\left(\boldsymbol{v}_{1}-\hat{\boldsymbol{u}}_{1}\right)\right\|_{0, K} \\
& \leq C \sigma^{-1} h|\boldsymbol{u}|_{1, \Omega}\left\|\pi\left(\boldsymbol{v}_{1}-\hat{\boldsymbol{u}}_{1}\right)\right\|_{0, \Omega}
\end{aligned}
$$

Collecting all the above results it follows from Lemma 1 that

$$
\frac{1}{2}\left\|\left(\pi\left(\boldsymbol{v}_{1}-\hat{\boldsymbol{u}}_{1}\right), q_{0}-\hat{p}_{0}\right)\right\|_{h}^{2} \leq C\left(\sqrt{\sigma} h\|\boldsymbol{u}\|_{2, \Omega}+\frac{h}{\sqrt{\sigma}}|p|_{1, \Omega}\right)\left\|\left(\pi\left(\boldsymbol{v}_{1}-\hat{\boldsymbol{u}}_{1}\right), \hat{p}_{0}-q_{0}\right)\right\|_{h}
$$

and hence (59) follows.

Next, we analyze the error in the $H^{d i v}(\Omega)$ norm for the velocity and the $L^{2}(\Omega)$ norm for the pressure. 
Theorem 8. Let $(\boldsymbol{u}, p)$ and $\left(\hat{\boldsymbol{u}}_{1}, \hat{p}_{0}\right)$ be the solutions of (3) and (16), respectively, and $\hat{\boldsymbol{u}}_{h}:=\pi\left(\hat{\boldsymbol{u}}_{1}\right)$. Under the hypothesis of Theorem 7 , there exists $C$ such that

$$
\begin{aligned}
\left\|\boldsymbol{u}-\hat{\boldsymbol{u}}_{h}\right\|_{d i v, \Omega} & \leq C h\left(\|\boldsymbol{u}\|_{2, \Omega}+\frac{1}{\sigma}|p|_{1, \Omega}\right), \\
\left\|\boldsymbol{u}-\hat{\boldsymbol{u}}_{h}-\ell\left(\llbracket \hat{p}_{0} \rrbracket\right)\right\|_{d i v, \Omega} & \leq C h\left(\|\boldsymbol{u}\|_{2, \Omega}+\frac{1}{\sigma}|p|_{1, \Omega}\right), \\
\left\|p-\hat{p}_{0}\right\|_{0, \Omega} & \leq C h\left(\sigma\|\boldsymbol{u}\|_{2, \Omega}+|p|_{1, \Omega}\right) .
\end{aligned}
$$

Proof. First, let $\boldsymbol{v}_{1}:=\mathcal{C}_{h}(\boldsymbol{u})$; then

$$
\begin{aligned}
\left\|\nabla \cdot\left(\boldsymbol{u}-\hat{\boldsymbol{u}}_{1}\right)\right\|_{0, \Omega}^{2} & =\left(\nabla \cdot\left(\boldsymbol{u}-\hat{\boldsymbol{u}}_{1}\right), \nabla \cdot\left(\boldsymbol{u}-\hat{\boldsymbol{u}}_{1}\right)\right)_{\Omega} \\
& =\left(\nabla \cdot\left(\boldsymbol{u}-\hat{\boldsymbol{u}}_{1}\right), \nabla \cdot\left(\boldsymbol{u}-\boldsymbol{v}_{1}\right)\right)_{\Omega}+\left(\nabla \cdot\left(\boldsymbol{u}-\hat{\boldsymbol{u}}_{1}\right), \nabla \cdot\left(\hat{\boldsymbol{u}}_{1}-\boldsymbol{v}_{1}\right)\right)_{\Omega} .
\end{aligned}
$$

Next, from Lemma 4 (considering $q_{0}:=\nabla \cdot\left(\hat{\boldsymbol{u}}_{1}-\boldsymbol{v}_{1}\right) \in Q_{h}$ and $\left.\boldsymbol{v}_{1}=\mathbf{0}\right)$ we get

$$
\begin{aligned}
\left(\nabla \cdot\left(\boldsymbol{u}-\hat{\boldsymbol{u}}_{1}\right), \nabla \cdot\left(\hat{\boldsymbol{u}}_{1}-\boldsymbol{v}_{1}\right)\right)_{\Omega} & =-\sum_{F \in \mathcal{E}_{h}} \tau_{F}\left(\llbracket p-\hat{p}_{0} \rrbracket, \llbracket \nabla \cdot\left(\hat{\boldsymbol{u}}_{1}-\boldsymbol{v}_{1}\right) \rrbracket\right)_{F} \\
& \leq \sum_{F \in \mathcal{E}_{h}} \tau_{F}\left\|\llbracket p-\hat{p}_{0} \rrbracket\right\|_{0, F}\left\|\llbracket \nabla \cdot\left(\hat{\boldsymbol{u}}_{1}-\boldsymbol{v}_{1}\right) \rrbracket\right\|_{0, F} \\
& \leq \sum_{F \in \mathcal{E}_{h}} \frac{\tau_{F}}{\gamma}\left\|\llbracket p-\hat{p}_{0} \rrbracket\right\|_{0, F}^{2}+\gamma \sum_{F \in \mathcal{E}_{h}} \tau_{F}\left\|\llbracket \nabla \cdot\left(\hat{\boldsymbol{u}}_{1}-\boldsymbol{v}_{1}\right) \rrbracket\right\|_{0, F}^{2} \cdot
\end{aligned}
$$

Next, using the local trace result (45), (42) and the mesh regularity to obtain

$$
\begin{aligned}
\gamma \sum_{F \in \mathcal{E}_{h}} \tau_{F}\left\|\llbracket \nabla \cdot\left(\hat{\boldsymbol{u}}_{1}-\boldsymbol{v}_{1}\right) \rrbracket\right\|_{0, F}^{2} & \leq C \gamma \sum_{K \in \mathcal{T}_{h}} \frac{\alpha h_{K}}{\sigma}\left[h_{K}^{-1}\left\|\nabla \cdot\left(\hat{\boldsymbol{u}}_{1}-\boldsymbol{v}_{1}\right)\right\|_{0, K}^{2}\right] \\
& \leq \frac{C \gamma}{\sigma} \sum_{K \in \mathcal{T}_{h}}\left[\left\|\nabla \cdot\left(\boldsymbol{u}-\hat{\boldsymbol{u}}_{1}\right)\right\|_{0, K}^{2}+\left\|\nabla \cdot\left(\boldsymbol{u}-\boldsymbol{v}_{1}\right)\right\|_{0, K}^{2}\right] \\
& \leq \frac{C \gamma}{\sigma} \sum_{K \in \mathcal{T}_{h}}\left[\left\|\nabla \cdot\left(\boldsymbol{u}-\hat{\boldsymbol{u}}_{1}\right)\right\|_{0, K}^{2}+C h_{K}^{2}|\boldsymbol{u}|_{2, \omega_{K}}^{2}\right] .
\end{aligned}
$$

Hence, choosing $\gamma=\frac{\sigma}{4 C}$ in (68) and using $a b \leq\left(a^{2} / 4\right)+b^{2}$, the mesh regularity and (42) again, (66) and (67) become

$$
\begin{aligned}
\left\|\nabla \cdot\left(\boldsymbol{u}-\hat{\boldsymbol{u}}_{1}\right)\right\|_{0, \Omega}^{2} \leq & \frac{1}{4}\left\|\nabla \cdot\left(\boldsymbol{u}-\hat{\boldsymbol{u}}_{1}\right)\right\|_{0, \Omega}^{2}+\left\|\nabla \cdot\left(\boldsymbol{u}-\boldsymbol{v}_{1}\right)\right\|_{0, \Omega}^{2} \\
& +\sum_{F \in \mathcal{E}_{h}} \frac{\tau_{F}}{\gamma}\left\|\llbracket p-\hat{p}_{0} \rrbracket\right\|_{0, F}^{2}+\frac{C \gamma}{\sigma} \sum_{K \in \mathcal{T}_{h}}\left[\left\|\nabla \cdot\left(\boldsymbol{u}-\hat{\boldsymbol{u}}_{1}\right)\right\|_{0, K}^{2}+C h_{K}^{2}|\boldsymbol{u}|_{2, \omega_{K}}^{2}\right] \\
\leq & C \sigma^{-1} \sum_{F \in \mathcal{E}_{h}} \tau_{F}\left\|\llbracket p-\hat{p}_{0} \rrbracket\right\|_{0, F}^{2}+C h^{2}|\boldsymbol{u}|_{2, \Omega}^{2}+\frac{1}{2}\left\|\nabla \cdot\left(\boldsymbol{u}-\hat{\boldsymbol{u}}_{1}\right)\right\|_{0, \Omega}^{2} \\
\leq & C \sigma^{-1}\left\|\left(\boldsymbol{u}-\hat{\boldsymbol{u}}_{h}, p-\hat{p}_{0}\right)\right\|_{h}^{2}+C h^{2}|\boldsymbol{u}|_{2, \Omega}^{2}+\frac{1}{2}\left\|\nabla \cdot\left(\boldsymbol{u}-\hat{\boldsymbol{u}}_{1}\right)\right\|_{0, \Omega}^{2},
\end{aligned}
$$


and the result follows by applying Theorem 7 and extracting the square root.

Now, we use the local mass conservation feature to prove (64). In fact, we get

$$
\int_{K} \nabla \cdot\left(\boldsymbol{u}-\hat{\boldsymbol{u}}_{1}-\ell_{K}\left(\llbracket \hat{p}_{0} \rrbracket\right)\right)=0
$$

and then, since $\left.\nabla \cdot\left(\hat{\boldsymbol{u}}_{1}+\ell_{K}\left(\llbracket \hat{p}_{0} \rrbracket\right)\right)\right|_{K} \in \mathbb{R}$, we end up with

$$
\begin{aligned}
\left\|\nabla \cdot\left(\boldsymbol{u}-\hat{\boldsymbol{u}}_{1}-\ell_{K}\left(\llbracket \hat{p}_{0} \rrbracket\right)\right)\right\|_{0, K}^{2} & =\left(\nabla \cdot\left(\boldsymbol{u}-\hat{\boldsymbol{u}}_{1}-\ell_{K}\left(\llbracket \hat{p}_{0} \rrbracket\right)\right), \nabla \cdot\left(\boldsymbol{u}-\hat{\boldsymbol{u}}_{1}-\ell_{K}\left(\llbracket \hat{p}_{0} \rrbracket\right)\right)\right)_{K} \\
& =\left(\nabla \cdot\left(\boldsymbol{u}-\hat{\boldsymbol{u}}_{1}-\ell_{K}\left(\llbracket \hat{p}_{0} \rrbracket\right)\right), \nabla \cdot(\boldsymbol{u}-\pi(\boldsymbol{u}))\right)_{K} \\
& \leq C h|\nabla \cdot \boldsymbol{u}|_{1, K} \| \nabla \cdot\left(\boldsymbol{u}-\hat{\boldsymbol{u}}_{1}-\ell_{K}\left(\llbracket \hat{p}_{0} \rrbracket\right) \|_{0, K} .\right.
\end{aligned}
$$

As seen in Lemma 2 we can prove that

$$
\left\|\ell\left(\llbracket \hat{p}_{0} \rrbracket\right)\right\|_{0, \Omega} \leq \sum_{F \in \mathcal{E}_{h}} \tau_{F}\left\|\boldsymbol{\varphi}_{F}\right\|_{0, \Omega}\left|\llbracket \hat{p}_{0} \rrbracket\right| \leq C h \sigma^{-\frac{1}{2}}\left\{\sum_{F \in \mathcal{E}_{h}} \tau_{F}\left\|\llbracket p-\hat{p}_{0} \rrbracket\right\|_{0, F}^{2}\right\}^{\frac{1}{2}}
$$

and then using (59) we obtain

$$
\left\|\boldsymbol{u}-\hat{\boldsymbol{u}}_{h}-\ell\left(\llbracket \hat{p}_{0} \rrbracket\right)\right\|_{0, \Omega} \leq C h\left(\|\boldsymbol{u}\|_{2, \Omega}+\frac{1}{\sigma}|p|_{1, \Omega}\right),
$$

and (64) follows from (70) and (72).

Finally, we consider the estimate for the pressure. From the continuous inf-sup condition (see [13]), there exists a $\boldsymbol{w} \in H_{0}^{1}(\Omega)$ such that $\nabla \cdot \boldsymbol{w}=p-\hat{p}_{0}$ in $\Omega$ and

$$
\|\boldsymbol{w}\|_{1, \Omega} \leq C\left\|p-\hat{p}_{0}\right\|_{0, \Omega}
$$

Let $\boldsymbol{w}_{1}=\mathcal{C}_{h}(\boldsymbol{w})$. Since $\nabla \cdot \boldsymbol{w}_{1}=\nabla \cdot\left(\pi\left(\boldsymbol{w}_{1}\right)\right)$, using Lemma 4 and recalling that $\pi_{K}=$ $\mathcal{I}-\sigma \mathcal{M}_{K}^{u}$, we obtain

$$
\begin{aligned}
\left\|p-\hat{p}_{0}\right\|_{0, \Omega}^{2}= & \left(\nabla \cdot \boldsymbol{w}, p-\hat{p}_{0}\right)_{\Omega} \\
= & \left(\nabla \cdot\left(\boldsymbol{w}-\boldsymbol{w}_{1}\right), p-\hat{p}_{0}\right)_{\Omega}+\left(\nabla \cdot \boldsymbol{w}_{1}, p-\hat{p}_{0}\right)_{\Omega} \\
= & \sum_{K \in \mathcal{T}_{h}}\left[\left(\boldsymbol{w}-\boldsymbol{w}_{1}, \nabla p\right)_{K}+\left(\boldsymbol{w}-\boldsymbol{w}_{1},\left(p-\hat{p}_{0}\right) \mathcal{I} \cdot \boldsymbol{n}\right)_{\partial K}\right] \\
& +\sum_{K \in \mathcal{T}_{h}} \sigma\left(\left(\mathcal{I}-\sigma \mathcal{M}_{K}^{u}\right)(\boldsymbol{u})-\pi_{K}\left(\boldsymbol{u}_{1}\right), \pi_{K}\left(\boldsymbol{w}_{1}\right)\right)_{K}+\sigma^{2}\left(\mathcal{M}_{K}^{u}(\boldsymbol{u}), \pi_{K}\left(\boldsymbol{w}_{1}\right)\right)_{K} \\
\leq & C\left[\sum_{K \in \mathcal{T}_{h}} h_{K}^{2}|p|_{1, K}^{2}+\sigma\left\|\left(\boldsymbol{u}-\pi\left(\boldsymbol{u}_{1}\right), p-\hat{p}_{0}\right)\right\|_{h}^{2}\right]^{\frac{1}{2}} \cdot \\
& {\left[\sum_{K \in \mathcal{T}_{h}} h_{K}^{-2}\left\|\boldsymbol{w}-\boldsymbol{w}_{1}\right\|_{0, K}^{2}+\sum_{F \in \mathcal{E}_{h}} h_{F}^{-1}\left\|\boldsymbol{w}-\boldsymbol{w}_{1}\right\|_{0, F}^{2}+\left\|\pi\left(\boldsymbol{w}_{1}\right)\right\|_{0, \Omega}^{2}\right]^{\frac{1}{2}} . }
\end{aligned}
$$


Now, using (42)-(43), the regularity of the mesh, (52) and (73) we obtain

$$
\left[\sum_{K \in \mathcal{T}_{h}} h_{K}^{-2}\left\|\boldsymbol{w}-\boldsymbol{w}_{1}\right\|_{0, K}^{2}+\sum_{F \in \mathcal{E}_{h}} \tau_{F}^{-1}\left\|\boldsymbol{w}-\boldsymbol{w}_{1}\right\|_{0, F}^{2}+\left\|\pi\left(\boldsymbol{w}_{1}\right)\right\|_{0, \Omega}^{2}\right]^{1 / 2} \leq C|\boldsymbol{w}|_{1, \Omega} \leq C\left\|p-\hat{p}_{0}\right\|_{0, \Omega},
$$

hence, dividing by $\left\|p-\hat{p}_{0}\right\|_{0, \Omega}$ and using Theorem 7 , we arrive at

$$
\left\|p-\hat{p}_{0}\right\|_{0, \Omega} \leq C\left[\sigma\left\|\left(\boldsymbol{u}-\pi\left(\hat{\boldsymbol{u}}_{1}\right), p-\hat{p}_{0}\right)\right\|_{h}^{2}+\sigma^{2} h^{2}|p|_{1, \Omega}^{2}\right]^{\frac{1}{2}} \leq C h\left[\sigma\|\boldsymbol{u}\|_{2, \Omega}+|p|_{1, \Omega}\right],
$$

and the result follows.

Remark. Having assumed the coefficient $\sigma$ to be constant in $\Omega$ (and then independent of any small scale), the $H^{2}(\Omega)$-norm of the exact solution $\boldsymbol{u}$ does not blow up as is usual in Darcy problems with highly oscillating coefficients (see [15] for further details).

3.3. An error estimate for the method (13). We end the error analysis by proving the following error result concerning the method (13).

Theorem 9. Let $(\boldsymbol{u}, p) \in H_{0}^{d i v}(\Omega) \cap H^{2}(\Omega)^{2} \times H^{1}(\Omega) \cap L_{0}^{2}(\Omega)$ be the solution of (2), and $\left(\boldsymbol{u}_{1}, p_{0}\right),\left(\hat{\boldsymbol{u}}_{1}, \hat{p}_{0}\right)$ the solution of methods (13) and (16), respectively. Then, defining $\boldsymbol{u}_{h}:=$ $\pi\left(\boldsymbol{u}_{1}\right)$ and $\hat{\boldsymbol{u}}_{h}:=\pi\left(\hat{\boldsymbol{u}}_{1}\right)$, the following error estimate holds

$$
\left\|\left(\boldsymbol{u}-\boldsymbol{u}_{h}, p-p_{0}\right)\right\|_{h} \leq 3\left\|\left(\boldsymbol{u}-\hat{\boldsymbol{u}}_{h}, p-\hat{p}_{0}\right)\right\|_{h} .
$$

Proof. First, from Lemmas 3 and 4 and Lemma 2 it follows

$$
\begin{aligned}
& \frac{1}{2}\left\|\left(\pi\left(\boldsymbol{u}_{1}-\hat{\boldsymbol{u}}_{1}\right), p_{0}-\hat{p}_{0}\right)\right\|_{h}^{2} \leq \mathbf{B}\left(\left(\boldsymbol{u}_{1}-\hat{\boldsymbol{u}}_{1}, p_{0}-\hat{p}_{0}\right),\left(\boldsymbol{u}_{1}-\hat{\boldsymbol{u}}_{1}, p_{0}-\hat{p}_{0}\right)\right) \\
&=\mathbf{B}\left(\left(\boldsymbol{u}-\hat{\boldsymbol{u}}_{1}, p-\hat{p}_{0}\right),\left(\boldsymbol{u}_{1}-\hat{\boldsymbol{u}}_{1}, p_{0}-\hat{p}_{0}\right)\right)+\sum_{K \in \mathcal{T}_{h}}\left(\sigma \mathcal{M}_{K}^{u}(\boldsymbol{u}), \sigma \pi_{K}\left(\boldsymbol{u}_{1}-\hat{\boldsymbol{u}}_{1}\right)\right)_{K} \\
&\left.=\mathbf{B}_{s}\left(\left(\boldsymbol{u}-\hat{\boldsymbol{u}}_{1}, p-\hat{p}_{0}\right),\left(\boldsymbol{u}_{1}-\hat{\boldsymbol{u}}_{1}, \hat{p}_{0}-p_{0}\right)\right)+\sum_{K \in \mathcal{T}_{h}}\left(\llbracket p-\hat{p}_{0} \rrbracket\right), \sigma \pi_{K}\left(\boldsymbol{u}_{1}-\hat{\boldsymbol{u}}_{1}\right)\right)_{K} \\
&+\sum_{K \in \mathcal{T}_{h}} \sigma\left(\mathcal{M}_{K}^{u}(\boldsymbol{u}), \sigma \pi_{K}\left(\boldsymbol{u}_{1}-\hat{\boldsymbol{u}}_{1}\right)\right)_{K} \\
&=\sum_{K \in \mathcal{T}_{h}}\left(\ell_{K}\left(\llbracket p-\hat{p}_{0} \rrbracket\right), \sigma \pi_{K}\left(\left(\boldsymbol{u}_{1}-\hat{\boldsymbol{u}}_{1}\right)\right)_{K}\right. \\
& \leq C_{1}\left\{\sum_{F \in \mathcal{E}_{h}} \tau_{F}\left\|\llbracket p-\hat{p}_{0} \rrbracket\right\|_{0, F}^{2}\right\}^{\frac{1}{2}} \sqrt{\sigma \alpha} h\left\|\pi\left(\boldsymbol{u}_{1}-\hat{\boldsymbol{u}}_{1}\right)\right\|_{0, \Omega} \\
& \leq C_{1}^{2} \alpha h^{2} \sum_{F \in \mathcal{E}_{h}} \tau_{F}\left\|\llbracket p-\hat{p}_{0} \rrbracket\right\|_{0, F}^{2}+\frac{1}{4} \sigma\left\|\pi\left(\boldsymbol{u}_{1}-\hat{\boldsymbol{u}}_{1}\right)\right\|_{0, \Omega}^{2} \\
& \leq\left\|\left(\boldsymbol{u}-\hat{\boldsymbol{u}}_{h}, p-\hat{p}_{0}\right)\right\|_{h}^{2}+\frac{1}{4}\left\|\left(\pi\left(\boldsymbol{u}_{1}-\hat{\boldsymbol{u}}_{1}\right), p_{0}-\hat{p}_{0}\right)\right\|_{0, \Omega}^{2}
\end{aligned}
$$


where we used $\alpha \leq \frac{1}{C_{1}^{2} h^{2}}$ and $C_{1}$ is the positive constant from Lemma 2. The result follows applying the triangular inequality.

Remark. We end this section by remarking that the same analysis from Theorem 8 may be carried out to prove error estimates on $\left\|\boldsymbol{u}-\hat{\boldsymbol{u}}_{h}\right\|_{d i v, \Omega}$ and $\left\|p-\hat{p}_{0}\right\|_{0, \Omega}$ as well.

\section{Numerical experiments}

Now, we are interested in the numerical validation of the PGEM in its symmetric version (16). The method (13) behaves similarly as shown in Section 3.3. Two numerical tests with available analytical solutions are performed and the theoretical results validated. The assumed vanishing boundary condition to generate the methods is adopted by the first numerical test, a property which is no longer shared by the second case. In all the computations the value for $\alpha_{F}$ has been set to one.

4.1. An analytical problem. The domain is $\Omega=(0,1) \times(0,1)$ and we set $\sigma=1$ and the exact pressure is given by $p(x, y)=\cos (2 \pi x) \cos (2 \pi y)$. Next, the exact velocity is determined from the Darcy law and the boundary condition is taken to be its normal component on the boundary, thus $b=0$. Consequently, the divergence velocity field is set as $g=8 \pi^{2} \cos (2 \pi x) \cos (2 \pi y)$.

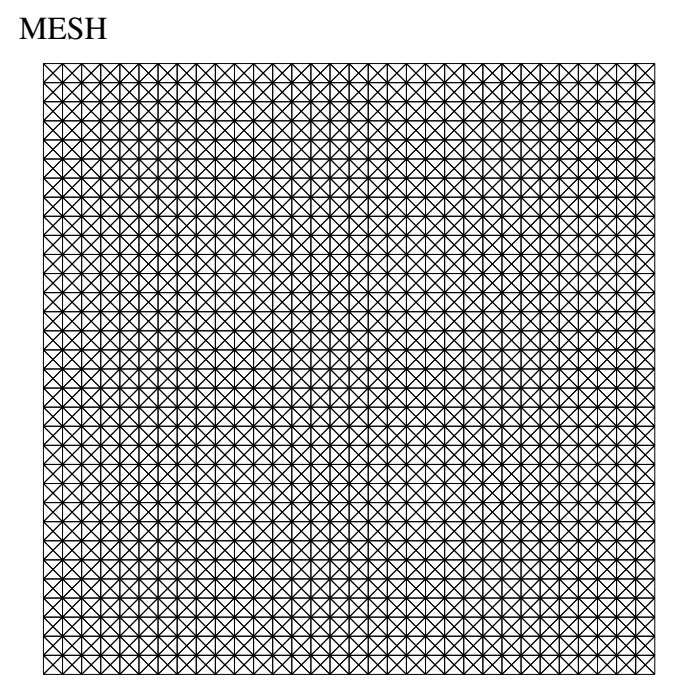

FIGURE 2. Mesh for the analytical problem.

In Figures 4-6 we report the errors on velocity and pressure in a sequence of structured meshes. One observes optimal convergence of all quantities as $h \rightarrow 0$ in their respective 
natural norms, which are in accordance with the theoretical results. Moreover, in Figure 4 we also plot the error $\left\|\pi(\boldsymbol{u})-\pi\left(\boldsymbol{u}_{1}\right)\right\|_{0, \Omega}$ which is smaller than $\left\|\boldsymbol{u}-\boldsymbol{u}_{1}\right\|_{0, \Omega}$. Here we denote $\left|\llbracket p-p_{0} \rrbracket\right|_{h}:=\left[\sum_{F \in \mathcal{E}_{h}} \tau_{F}\left\|\llbracket p-p_{0} \rrbracket\right\|_{0, F}^{2}\right]^{1 / 2}$.

First, we adopt the structured mesh described in Figure 2 which contains 4096 triangular elements $\left(h=3.125 \times 10^{-2}\right)$. We depict in Figure 3 the isolines (free of oscillations) of the pressure and $\left|\boldsymbol{u}_{1}\right|$ obtained from (16).

Furthermore, in Table 4.1 we study the local mass conservation feature of (16) whether we look at either $\boldsymbol{u}_{1}$ or $\boldsymbol{u}_{1}+\boldsymbol{u}_{e}$. We define the quantities

$$
M_{e}:=\max _{K \in \mathcal{T}_{h}} \frac{\left|\int_{K}\left(\nabla \cdot\left(\boldsymbol{u}_{1}+\boldsymbol{u}_{e}\right)-g\right) d \mathbf{x}\right|}{|K|} \quad \text { and } \quad M_{1}:=\max _{K \in \mathcal{T}_{h}} \frac{\left|\int_{K}\left(\nabla \cdot \boldsymbol{u}_{1}-g\right) d \mathbf{x}\right|}{|K|}
$$

and we find a loss of mass, as expected, when just the linear part of the solution is used. Nevertheless, we recover the local mass conservation property updating the linear velocity field by the multi-scale velocity $\boldsymbol{u}_{e}$. Similar results were obtained in [7] using the nonsymmetric PGEM (32).

\begin{tabular}{|c|c|c|c|c|c|c|}
\hline$h$ & 0.25 & 0.125 & $6.25 \times 10^{-2}$ & $3.125 \times 10^{-2}$ & $1.5625 \times 10^{-2}$ & $7.8125 \times 10^{-3}$ \\
\hline$M_{e}$ & $2.3 \times 10^{-14}$ & $2.3 \times 10^{-13}$ & $10^{-12}$ & $10^{-11}$ & $1.2 \times 10^{-10}$ & $10^{-9}$ \\
\hline$M_{1}$ & 0.81 & 0.35 & 0.09 & 0.03 & $6.4 \times 10^{-3}$ & $1.6 \times 10^{-3}$ \\
\hline
\end{tabular}

Table 4.1: Relative local mass conservation errors with the symmetric method (16). 
PRESSURE

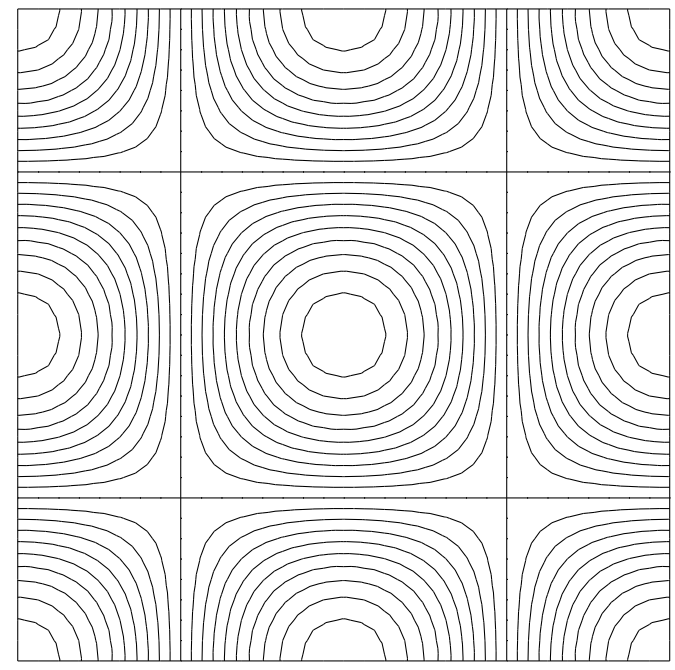

\section{VELOCITY}

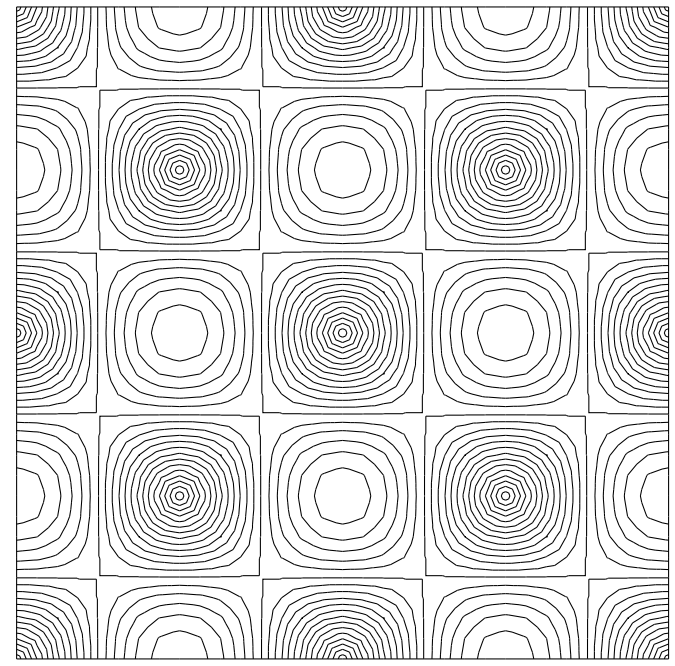

Figure 3. Isolines of the pressure (left) and $\left|\boldsymbol{u}_{1}\right|$ (right) using the symmetric method (16).

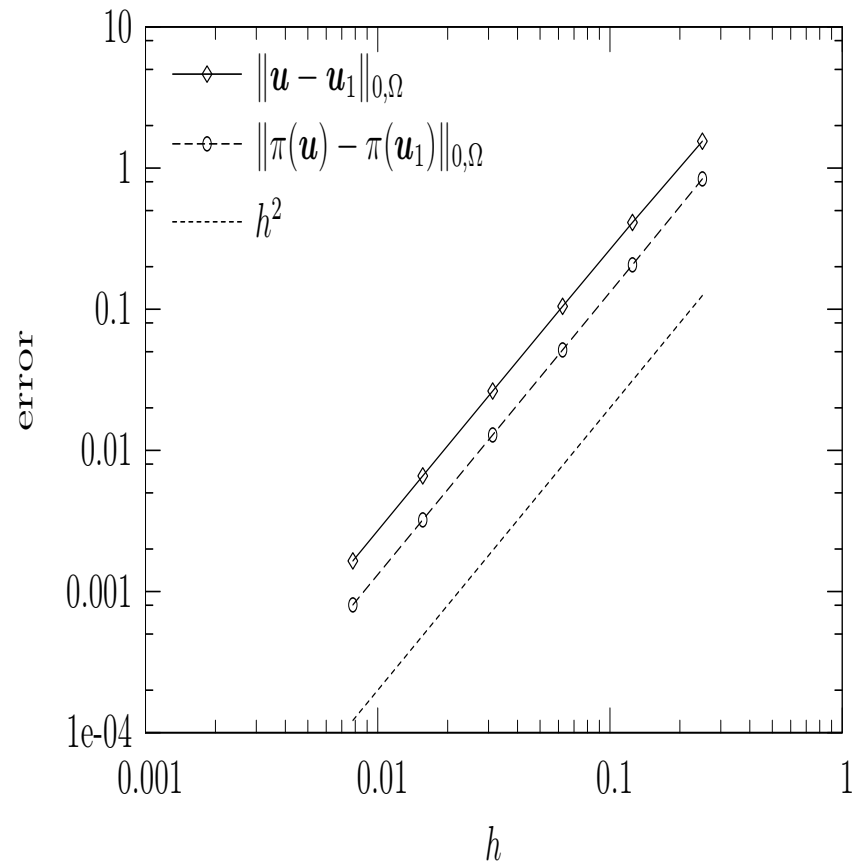

Figure 4. Convergence history of $\left\|\boldsymbol{u}-\boldsymbol{u}_{1}\right\|_{0, \Omega}$ and $\left\|\pi(\boldsymbol{u})-\pi\left(\boldsymbol{u}_{1}\right)\right\|_{0, \Omega}$ for the symmetric method (16). 

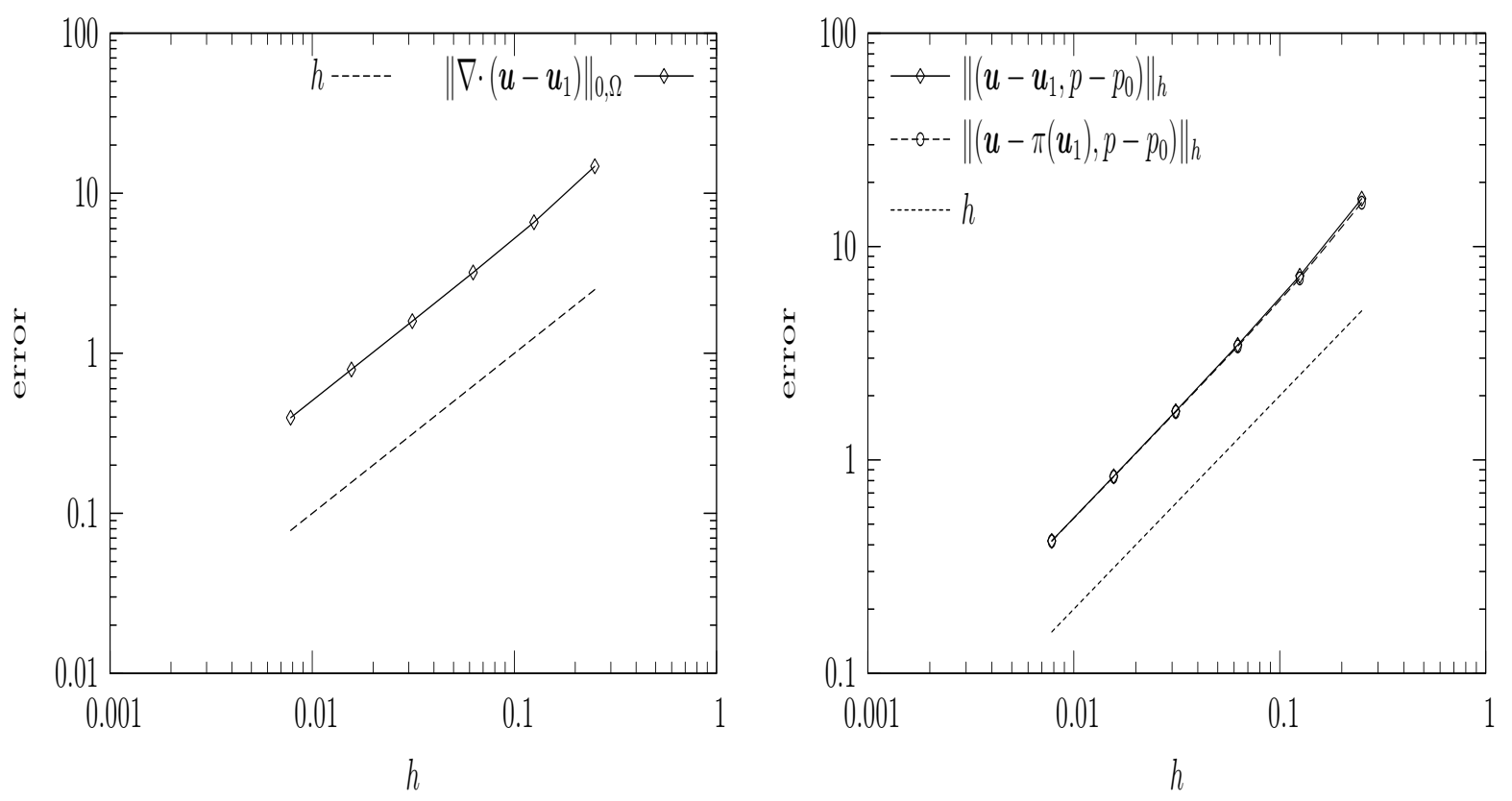

Figure 5. Convergence history of $\left\|\nabla \cdot\left(\boldsymbol{u}-\boldsymbol{u}_{1}\right)\right\|_{0, \Omega}$ and $\left\|\left(\boldsymbol{u}-\boldsymbol{u}_{1}, p-p_{0}\right)\right\|_{h}$, and $\left\|\left(\boldsymbol{u}-\pi\left(\boldsymbol{u}_{1}\right), p-p_{0}\right)\right\|_{h}$ for the symmetric method (16).
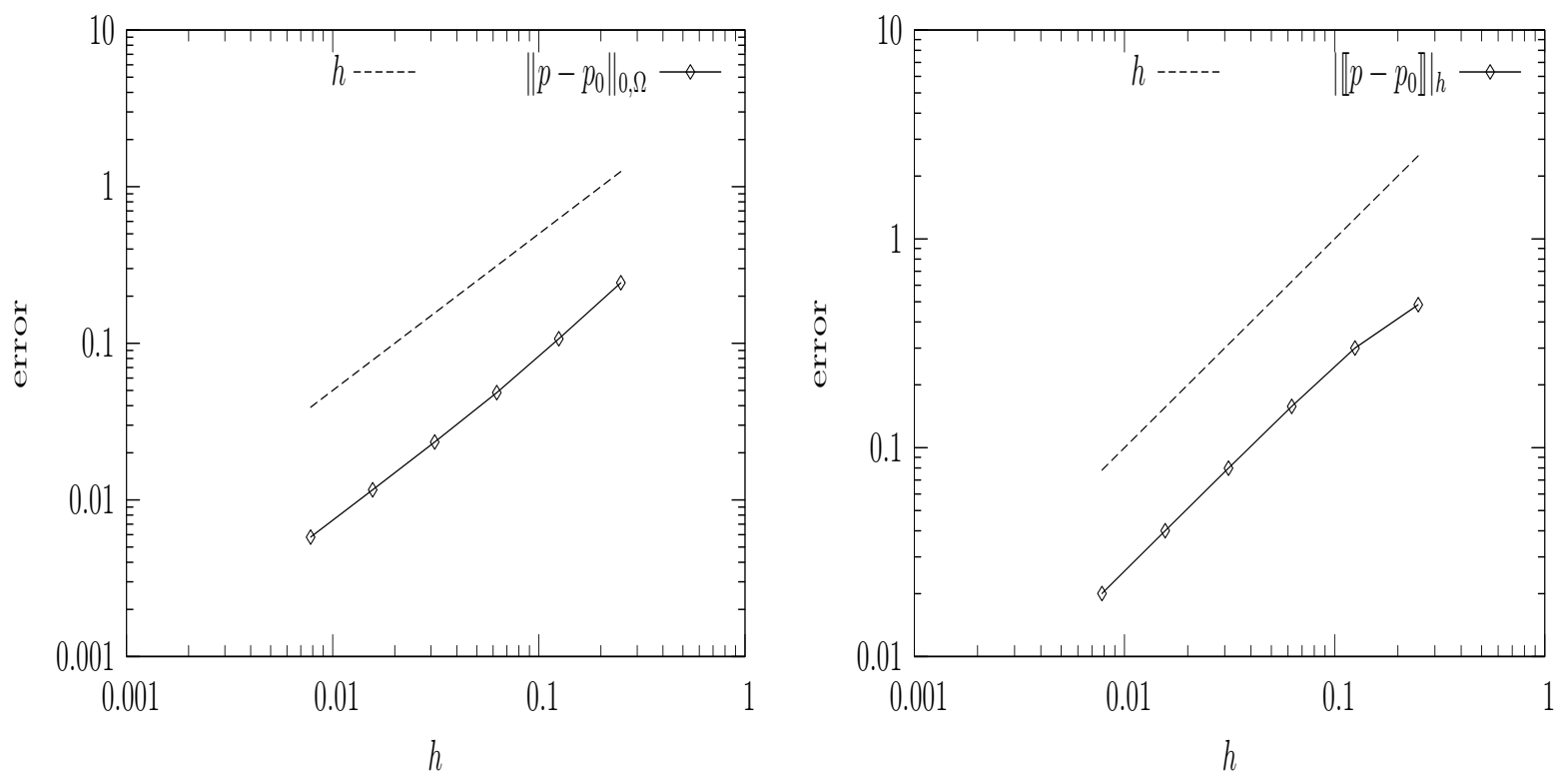

Figure 6 . Convergence history of $\left\|p-p_{0}\right\|_{0, \Omega}$ and $\left|\llbracket p-p_{0} \rrbracket\right|_{h}$ for the symmetric method (16). 
4.2. A second analytical problem. The problem is set up as in the first test, differing by replacing the previous exact pressure by $p(x, y)=\frac{x^{3} y}{3}-\frac{y^{3} x}{3}$. Once again, the velocity is computed from the Darcy law and the boundary condition $b$ is taken to be its no vanishing normal component on the boundary. On the other hand, clearly the velocity field is divergence free $(g=0)$.

We validate the symmetric method (16) using a sequence of structured meshes. Optimality is reached whatever the norm is considered as shown in Figures 8-10. Similar quadratic convergence is observed for $\left\|\boldsymbol{u}-\boldsymbol{u}_{1}\right\|_{0, \Omega}$ and $\left\|\pi(\boldsymbol{u})-\pi\left(\boldsymbol{u}_{1}\right)\right\|_{0, \Omega}$. Table 4.2 highlights that the local mass conservation property is recovered as soon as $\boldsymbol{u}_{1}$ is updated by $\boldsymbol{u}_{1}+\boldsymbol{u}_{e}$.

\begin{tabular}{|c|c|c|c|c|c|c|}
\hline$h$ & 0.25 & 0.125 & $6.25 \times 10^{-2}$ & $3.125 \times 10^{-2}$ & $1.5625 \times 10^{-2}$ & $7.8125 \times 10^{-3}$ \\
\hline$M_{e}$ & $3.1 \times 10^{-15}$ & $1.5 \times 10^{-14}$ & $1.9 \times 10^{-13}$ & $7.1 \times 10^{-13}$ & $5.6 \times 10^{-12}$ & $7.6 \times 10^{-11}$ \\
\hline$M_{1}$ & 0.14 & 0.08 & 0.04 & 0.02 & 0.01 & $5.3 \times 10^{-3}$ \\
\hline
\end{tabular}

Table 4.2: Relative local mass conservation errors with the symmetric method (16).

Next, the structured mesh of Figure 2 is once more adopted, and the solution is oscillationfree as it can be seen through the isolines of $p_{0}$ and $\boldsymbol{u}_{1}$ in Figure 7.

PRESSURE

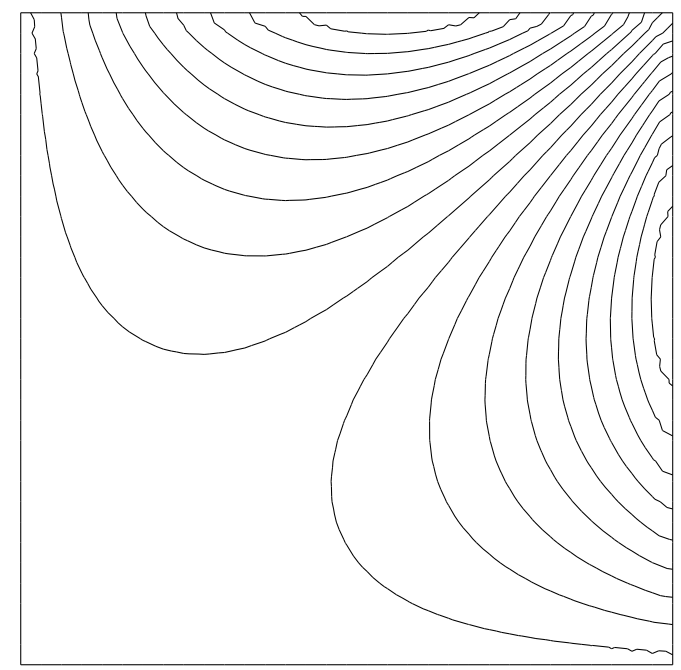

\section{VELOCITY}

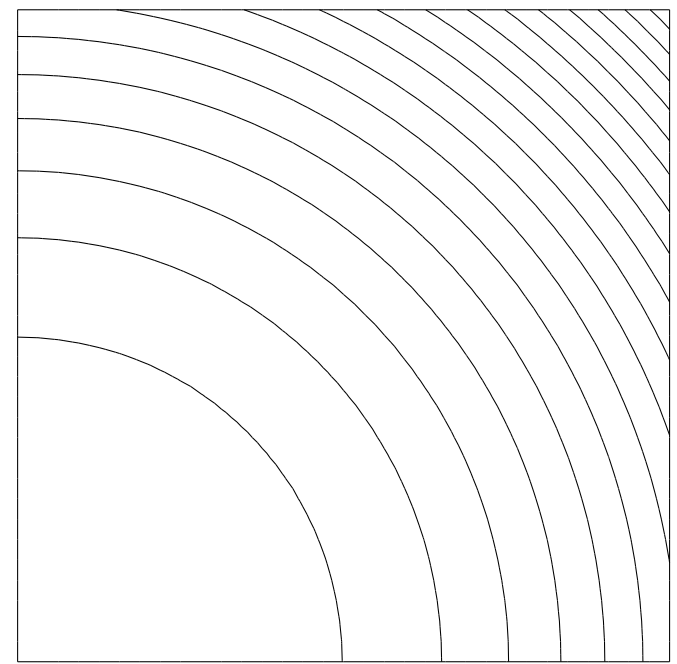

FiguRE 7. Isolines of the pressure (left) and $\left|\boldsymbol{u}_{1}\right|$ (right) using the symmetric method (16). 


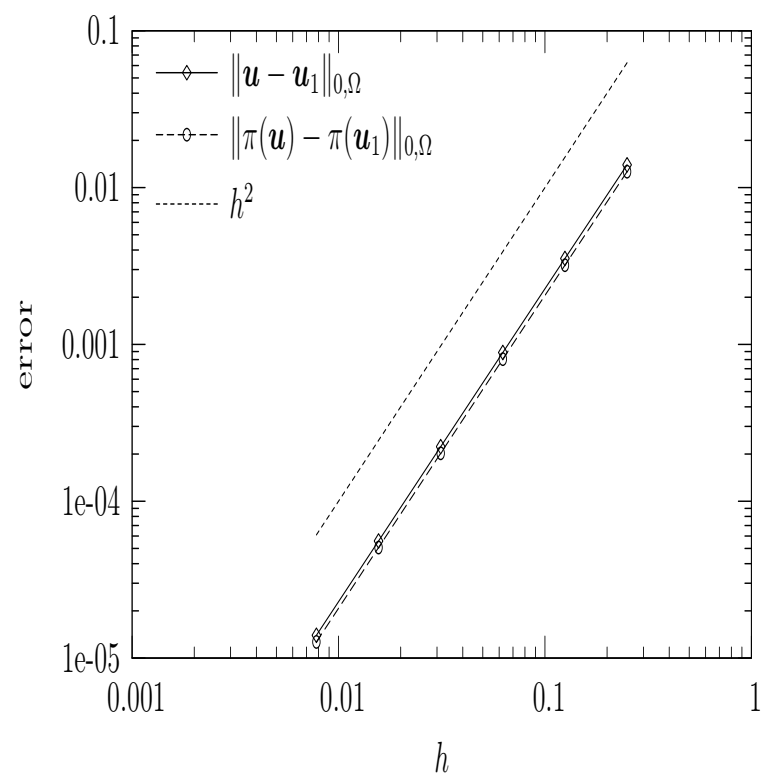

Figure 8. Convergence history of $\left\|\boldsymbol{u}-\boldsymbol{u}_{1}\right\|_{0, \Omega}$ and $\left\|\pi(\boldsymbol{u})-\pi\left(\boldsymbol{u}_{1}\right)\right\|_{0, \Omega}$ for the symmetric method (16).
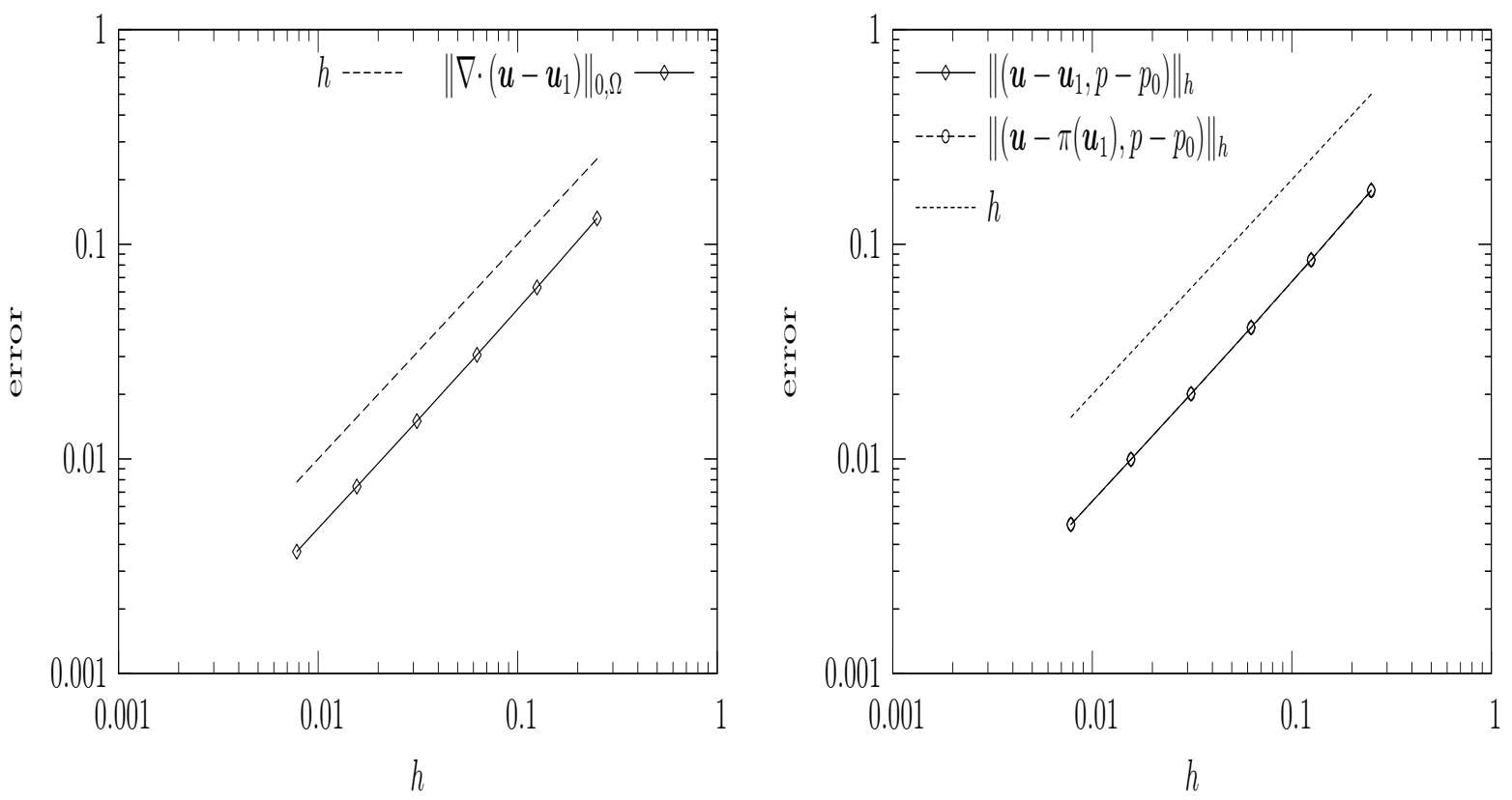

Figure 9. Convergence history of $\left\|\nabla \cdot\left(\boldsymbol{u}-\boldsymbol{u}_{1}\right)\right\|_{0, \Omega}$ and $\left\|\left(\boldsymbol{u}-\boldsymbol{u}_{1}, p-p_{0}\right)\right\|_{h}$, and $\left\|\left(\boldsymbol{u}-\pi\left(\boldsymbol{u}_{1}\right), p-p_{0}\right)\right\|_{h}$ for the symmetric method (16). 

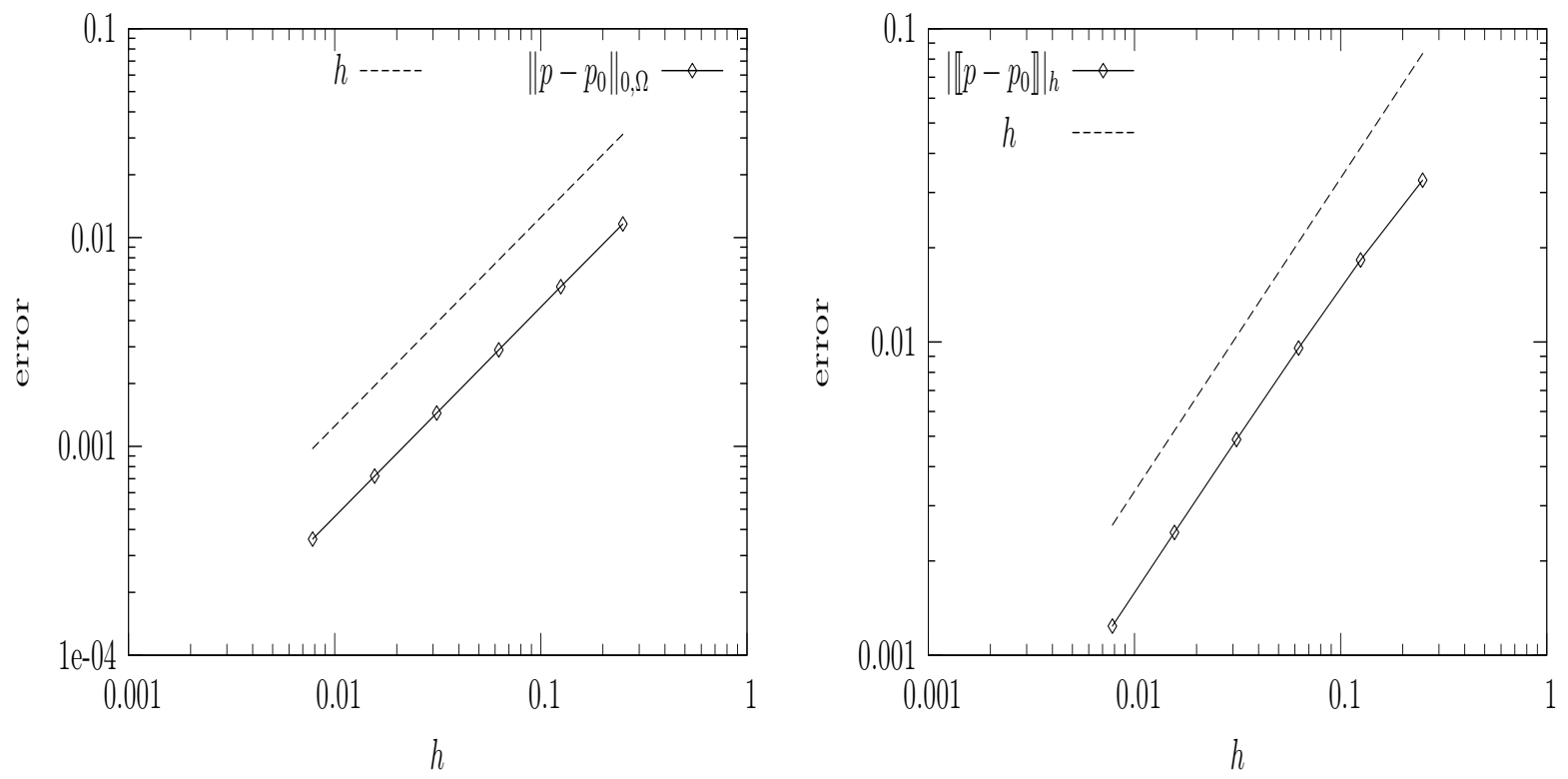

Figure 10. Convergence history of $\left\|p-p_{0}\right\|_{0, \Omega}$ and $\left|\llbracket p-p_{0} \rrbracket\right|_{h}$ for the symmetric method (16).

\section{Conclusion}

New enriched finite element methods make the simplest pair of nodal based interpolation spaces stable for the Darcy model. It has been proved that such methods lead to optimal error estimates in natural norms in addition to be locally mass conservative. Such fundamental property is recovered inside a general framework which relies on updating the linear part of velocity with a particular Raviart-Thomas function. Such strategy can prevent other jump-based stabilized finite element methods from local loss of mass while keeping them stable and accurate. Alternatives to deal with higher order interpolations should include additional control on the jumps of gradient of the pressure, in the form of a new enrichment function leading to a term like $\left(\ell\left(\llbracket \nabla p_{1} \cdot \boldsymbol{n} \rrbracket\right), \nabla q_{1}\right)_{\Omega}$, a feature that may be incorporated into the current Petrov-Galerkin framework.

\section{Appendix A. The error for general $g$}

If we do not suppose that $g$ is a piecewise constant function, instead we admit that $g \in H^{1}(\Omega)$, then a third enrichment function $\left(\boldsymbol{u}_{e}^{g}, p_{e}^{g}\right)$ appears as the solution of

$$
\begin{gathered}
\sigma \boldsymbol{u}_{e}^{g}+\nabla p_{e}^{g}=\mathbf{0} \quad \text { in } K, \quad \nabla \cdot \boldsymbol{u}_{e}^{g}=g-\Pi_{K}(g) \quad \text { in } K, \\
\boldsymbol{u}_{e}^{g} \cdot \boldsymbol{n}=0 \quad \text { in } \partial K
\end{gathered}
$$


Now, denoting $\left(\boldsymbol{u}_{e}^{g}, p_{e}^{g}\right)=\mathcal{G}_{K}\left(g-\Pi_{K}(g)\right)$, then, the original method (13) becomes: Find $\left(\tilde{\boldsymbol{u}}_{1}, \tilde{p}_{0}\right) \in \mathbf{V}_{h} \times Q_{h}$ such that for all $\left(\boldsymbol{v}_{1}, q_{0}\right) \in \mathbf{V}_{h} \times Q_{h}$ it holds

$$
\mathbf{B}\left(\left(\tilde{\boldsymbol{u}}_{1}, \tilde{p}_{0}\right),\left(\boldsymbol{v}_{1}, q_{0}\right)\right)=\mathbf{F}_{s}\left(\pi\left(\boldsymbol{v}_{1}\right), q_{0}\right)-\sum_{K \in \mathcal{T}_{h}}\left(\mathcal{G}_{K}\left(g-\Pi_{K}(g)\right), \sigma \pi_{K}\left(\boldsymbol{v}_{1}\right)\right)_{K},
$$

where we recall that $\Pi_{K}(g)=\frac{1}{|K|} \int_{K} g$.

Remark. Regarding the symmetric method its right hand side must be enhanced with the same contribution as well, and then the following results also apply to such version.

Remark. Next, we see that there exists $C>0$ such that

$$
\left\|\boldsymbol{u}_{e}^{g}\right\|_{0, K} \leq C h_{K}\left\|g-\Pi_{K}(g)\right\|_{0, K} \quad \forall K \in \mathcal{T}_{h}
$$

Indeed, we remark that multiplying $(77)$ by $\boldsymbol{u}_{e}^{g}$ and integrating it by parts we obtain that

$$
\begin{aligned}
\left\|\boldsymbol{u}_{e}^{g}\right\|_{0, K}^{2} & =-\frac{1}{\sigma}\left(\nabla p_{e}^{g}, \boldsymbol{u}_{e}^{g}\right)_{K} \\
& =\frac{1}{\sigma}\left(p_{e}^{g}, \nabla \cdot \boldsymbol{u}_{e}^{g}\right)_{K} \\
& =\frac{1}{\sigma}\left(p_{e}^{g}, g-\Pi_{K}(g)\right)_{K} \\
& \leq \frac{1}{\sigma}\left\|p_{e}^{g}\right\|_{0, K}\left\|g-\Pi_{K}(g)\right\|_{0, K} \\
& \leq C \frac{h_{K}}{\sigma}\left|p_{e}^{g}\right|_{1, K}\left\|g-\Pi_{K}(g)\right\|_{0, K} \\
& =C h_{K}\left\|\boldsymbol{u}_{e}^{g}\right\|_{0, K}\left\|g-\Pi_{K}(g)\right\|_{0, K},
\end{aligned}
$$

and the result follows.

Now, applying the Strang Lemma (cf. [17]) and (78) we arrive at

$$
\begin{aligned}
\left\|\left(\pi\left(\tilde{\boldsymbol{u}}_{1}-\boldsymbol{u}_{1}\right), \tilde{p}_{0}-p_{0}\right)\right\|_{h} & \leq \sup _{\left(\boldsymbol{v}_{1}, q_{0}\right) \in \mathbf{V}_{h} \times Q_{h}^{0}-\{0\}} \frac{-\sum_{K \in \mathcal{T}_{h}}\left(\mathcal{G}_{K}\left(g-\Pi_{K}(g)\right), \sigma \pi_{K}\left(\boldsymbol{v}_{1}\right)\right)_{K}}{\left\|\left(\pi\left(\boldsymbol{v}_{1}\right), q_{0}\right)\right\|_{h}} \\
& \leq C\left[\sum_{K \in \mathcal{T}_{h}}\left\|\mathcal{G}_{K}\left(g-\Pi_{K}(g)\right)\right\|_{0, K}^{2}\right]^{\frac{1}{2}} \\
& \leq C h\left\|g-\Pi_{K}(g)\right\|_{0, \Omega} \\
& \leq C h^{2}|g|_{1, \Omega},
\end{aligned}
$$

and then, as claimed, we see that the error is not affected by the fact that we projected $g$ onto the piecewise constant space. Therefore, following the same strategy in $\S 3$ we can 
estimate pressure and divergence of velocity in their respective natural norms, and so they are summarized in the following theorem:

Theorem 10. Let us suppose that $g \in H^{1}(\Omega)$ and denote $\tilde{\boldsymbol{u}}_{h}:=\pi\left(\tilde{\boldsymbol{u}}_{1}\right)$. Then, under the hypothesis of Theorem 9, the following error estimates hold

$$
\begin{aligned}
\left\|\boldsymbol{u}-\tilde{\boldsymbol{u}}_{h}\right\|_{d i v, \Omega} & \leq C h\left(|\boldsymbol{u}|_{2, \Omega}+\frac{1}{\sigma}|p|_{1, \Omega}+h|g|_{1, \Omega}\right), \\
\left\|\boldsymbol{u}-\tilde{\boldsymbol{u}}_{h}-\ell\left(\tilde{p}_{0}\right)\right\|_{d i v, \Omega} & \leq C h\left(|\boldsymbol{u}|_{2, \Omega}+\frac{1}{\sigma}|p|_{1, \Omega}+h|g|_{1, \Omega}\right), \\
\left\|p-\tilde{p}_{0}\right\|_{0, \Omega} & \leq C h\left(\sigma|\boldsymbol{u}|_{2, \Omega}+|p|_{1, \Omega}+\sigma h|g|_{1, \Omega}\right) .
\end{aligned}
$$

Acknowledgments. A part of this work was performed during the stay of Gabriel Barrenechea at the Applied Mathematics Dpt., LNCC, Brazil, in the framework of joint Chile(CONICYT)-Brazil(CNPq) project No. 2005-073 (Chile)-490639/2005-4 (Brazil). The authors want to thank Mark Ainsworth and Rodolfo Rodríguez for helpful discussions and comments.

\section{REFERENCES}

[1] J. E. Aarnes And Y. Efendiev, Mixed multiscale finite element methods for stochastic porous media flows, SIAM J. Sci. Comput., 30 (2008), pp. 2319-2339.

[2] R. Araya, G. R. Barrenechea, L. P. Franca, and F. Valentin, Stabilization arising from PGEM: A review and further developments, Applied Numerical Mathematics, 227 (2009), pp. 93-101.

[3] R. Araya, G. R. Barrenechea, and F. Valentin, Stabilized finite element methods based on multiscale enrichment for the Stokes problem, SIAM J. Numer. Anal., 44 (2006), pp. 322-348.

[4] R. Araya and F. Valentin, A multiscale a posteriori error estimate, Comput. Methods Appl. Mech. Engrg., 194 (2005), pp. 2077-2094.

[5] T. Arbogast, Analysis of a two-scale locally conservative subgrid upscaling for elliptic problems, SIAM J. Numer. Anal., 42 (2004), pp. 576-598.

[6] T. Arbogast and M. F. Wheeler, A family of rectangular mixed elements with a continuos flux for second order elliptic problems, SIAM J. Numer. Anal., 42 (2005), pp. 1914-1931.

[7] G. R. Barrenechea, L. P. Franca, and F. Valentin, A Petrov-Galerkin enriched method: a mass conservative finite element method for the Darcy equation, Computer Methods in Applied Mechanics and Engineering, 196 (2007), pp. 2449-2464.

[8] G. R. Barrenechea and F. Valentin, Relationship between multiscale enrichment and stabilized finite element methods for the generalized Stokes problem, CRAS, 341 (2005), pp. 635-640.

[9] C. Bernardi, T. Chacón-Rebollo, F. Hecht, and Z. Mghazli, Mortar finite element discretization of a model coupling Darcy and Stokes equations, M2AN, 42 (2008), pp. 375-410.

[10] P. Bochev and M. Gunzburger, On least-squares finite element methods for the Poisson equation and their connection to the Dirichlet and Kelvin principles, SIAM J. Numer. Anal., 42 (2005), pp. 340362 . 
[11] _ - A locally conservative least-squares method for Darcy flows, Communications in Numerical Methods in Engineering, 24 (2008), pp. 97-110.

[12] F. Brezzi, J. Douglas, And L. Marini, Two families of mixed finite elements for second order elliptic problems, Numer. Math., 47 (1985), pp. 217-235.

[13] F. Brezzi And M. Fortin, Mixed and Hybrid Finite Element Methods, vol. 15 of Springer Series in Computational Mathematics, Springer-Verlag, Berlin, New-York, 1991.

[14] E. Burman And P. Hansbo, A unified stabilized method for Stokes' and Darcy's equations, J. Comput. Appl. Math., 198 (2007), pp. 35-51.

[15] Z. Chen And T. Hou, A mixed multiscale finite element method for elliptic problems with oscillating coefficients, Mathematics of Computation, 72 (2002), pp. 541-576.

[16] P. ClÉMent, Approximation by finite element functions using local regularization, RAIRO Anal. Numér., (1975), pp. 77-84.

[17] A. Ern and J.-L. Guermond, Theory and Practice of Finite Elements, Springer-Verlag, 2004.

[18] L. P. Franca, A. L. Madureira, L. Tobiska, And F. Valentin, Convergence analysis of a multiscale finite element method for singularly perturbed problems, SIAM Multiscale Model. and Simul., 4 (2005), pp. 839-866.

[19] L. P. Franca, A. L. Madureira, and F. Valentin, Towards multiscale functions: enriching finite element spaces with local but not bubble-like functions, Comput. Methods Appl. Mech. Engrg., 194 (2005), pp. 3006-3021.

[20] L. P. Franca, J. V. A. Ramalho, and F. Valentin, Multiscale and Residual-Free Bubble functions for reaction-advection-diffusion Problems, International Journal for Multiscale Enginnering, 3 (2005), pp. 297-312.

[21] — Enriched finite element methods for unsteady reaction-diffusion problems, Communications in Numerical Methods in Engineering, 22 (2006), pp. 619-625.

[22] V. Girault and P. Raviart, Finite Element Methods for Navier-Stokes Equations: Theory and Algorithms, vol. 5 of Springer Series in Computational Mathematics, Springer-Verlag, Berlin, New-York, 1986.

[23] T. J. R. Hughes, A. Masud, And J. Wan, A stabilized mixed discontinuos Galerkin method for Darcy flow, Comput. Methods Appl. Mech. Engrg., 195 (2006), pp. 3347-3381.

[24] J. Li, T. Arbogast, and Y. Huang, Mixed methods using standard conforming finite elements, Comput. Methods Appl. Mech. Engrg., 198 (2009), pp. 680-692.

[25] K. Mardal, X. Tai, And R. Winther, A robust finite element method for Darcy-Stokes flow, SIAM J. Numer. Anal., 40 (2002), pp. 1605-1631.

[26] A. Masud and T. J. R. Hughes, A stabilized mixed finite element method for Darcy flow, Comput. Methods Appl. Mech. Engrg., (2002), pp. 4341-4370.

[27] R. Raviart and J. Thomas, A mixed finite element method for 2nd order elliptic problems, Mathematical aspect of finite element methods, no. 606 in Lecture Notes in Mathematics, Springer-Verlag, New York, 1977, pp. 292-315. 
Department of Mathematics, University of Strathclyde, 26 Richmond Street, Glasgow G1 1XH, UK

E-mail address: grb@maths.strath.ac.uk

Department of Mathematical Sciences, University of Colorado at Denver, P.O. Box 173364, Campus Box 170 Denver, Colorado 80217-3364, USA

E-mail address: leo.franca@cudenver.edu

Departamento de Matemática Aplicada, laboratório Nacional de ComputaÇão Científica, Av. Getúlio Vargas, 333, 25651-070 Petrópolis - RJ, Brazil

E-mail address: valentin@lncc.br 